NATHAN EDUARDO RIBEIRO BATISTA

\title{
PREVISÃO E SIMULAÇÃO EM MODELOS DE EQUILÍBRIO GERAL DINÂMICO
}


NATHAN EDUARDO RIBEIRO BATISTA

\section{PREVISÃO E SIMULAÇÃO EM MODELOS DE EQUILÍBRIO GERAL DINÂMICO}

Dissertação apresentada à Escola Politécnica da Universidade de São Paulo para obtenção do Título de Mestre em Engenharia Elétrica. 


\section{PREVISÃO E SIMULAÇÃO EM MODELOS DE EQUILÍBRIO GERAL DINÂMICO}

Dissertação apresentada à Escola Politécnica da Universidade de São Paulo para obtenção do Título de Mestre em Engenharia Elétrica.

Área de Concentração:

Sistemas Eletrônicos

Orientador:

Prof. Livre Docente Flavio Magalhães Cipparrone 
Este exemplar foi revisado e corrigido em relação à versão original, sob responsabilidade única do autor e com a anuência de seu orientador.

São Paulo, de de

Assinatura do autor:

Assinatura do orientador:

Catalogação-na-publicação

Batista, Nathan Eduardo Ribeiro

Previsão e simulação em modelos de equilíbrio geral dinâmico / N. E. R.

Batista -- versão corr. -- São Paulo, 2015.

$60 \mathrm{p}$.

Dissertação (Mestrado) - Escola Politécnica da Universidade de São Paulo. Departamento de Engenharia de Sistemas Eletrônicos.

1.Modelos Matemáticos 2.Sistemas dinâmicos I.Universidade de São Paulo. Escola Politécnica. Departamento de Engenharia de Sistemas Eletrônicos II.t. 
Dedicatória

Aos meu pais Manuel in memoriam e Juracy.

À Ana Paula e Julia, que me fazem sonhar. 


\section{AGRADECIMENTOS}

Agradeço ao valioso suporte oferecido pelo Professor Livre Docente Flavio Magalhães Cipparrone em todos os momentos de elaboração deste trabalho e por suas ricas contribuições.

As minhas palavras de apreço e gratidão aos meus pais pelos exemplos e incentivos a mim oferecidos ao longo da vida. A perda muito recente do meu pai faz com que este trabalho venha a ser lembrado de uma forma especial. Afinal, ele sempre se apresentou como meu incentivador incansável que jamais mediu esforços, não obstante os obstáculos vividos, a fim de nos oferecer plena oferta de ferramentas de capacitação intelectual. Seus exemplos de determinação, persistência e cordialidade são os legados de papai que estão gravados de forma indelével nos registros do meu coração.

Minha gratidão a Ana Paula e Julia, que dia a dia se mostram como minhas pacientes e vigorosas apoiadoras de uma grande travessia.

O meu agradecimento ao meu amigo, incentivador, ideólogo dos temas quantitativos e brilhante autoridade em matemática aplicada, Roberto Masaishi, que sempre esteve disposto em auxiliar-me.

A leitura atenta e os refinados comentários recebidos dos brilhantes professores Klênio Barbosa e Daniel Monte, foi para mim uma brisa de novas idéias com efeito transformador. Registro toda a minha gratidão a estes gentlemen, - destacados membros da academia.

Os ensinos acerca dos modelos DSGE, desde a concepção inicial até os modelos estado da arte, que obtive junto ao economista Marcelo Toledo, autoridade no tema, foram-me de valor ímpar. Certamente tais ensinos permitiram-me dar os primeiros passos neste tão vasto e envolvente tema.

Agradeço a Deus pelo fôlego de vida. 
"Sertão que se alteia e abaixa, mas é o da vida"

-- Guimarães Rosa 


\section{RESUMO}

O objetivo deste trabalho é estudar as principais características de uma família de modelos que busca retratar a dinâmica do equilíbrio geral de uma economia. Por equilíbrio em economia entende-se como sendo uma situação onde nenhum agente econômico tem qualquer incentivo para modificar a sua estratégia escolhida. Neste trabalhamos não haverá uma preocupação em demonstrar a existência, unicidade e estabilidade do equilíbrio, mas sim entender como alterações exógenas afetam o equilíbrio.

Brevemente, podemos entender a definição de equilíbrio geral como sendo a perspectiva de análise de equilíbrio simultâneo entre todos os mercados de uma economia. No conceito de equilíbrio geral todos os mercados devem estar em equilíbrio, de modo que nenhuma mudança em um mercado específico não venha a recompensar um determinado agente. O equilíbrio geral é constratado com a perspectiva de equilíbrio parcial, no qual parte da economia é considerada, tomando-se como dado o que está acontecendo nos demais mercados.

Os modelos de equilíbrio geral que envolvem dinâmica e choques estocástico são conhecidos pelo acrônimo DSGE, ou seja, Dynamic Stochastic General Equilibrium. Na realidade trata-se de uma família de modelos, pois compreendem uma gama variada de modelos com diferentes níveis de sofisticação. Neste exercício pelo caráter generalista da modelagem decidimos por estudar o modelo DSGE proposto por Smets-Wouters (2003).

Veremos neste estudo uma descrição deste modelo, seus fundamentos teóricos, e uma simulação deste modelo, de modo a vermos quais as direções de política econômica que tal modelo nos permite compreender.

Os resultados obtidos utilizando-se dados da economia americana nos permitiu inferir direções para as variáveis produto e consumo diante de um choque de inovação. Foi possível por meio do modelo adotado estimar uma direção para a trajetória do PIB para os próximos anos.

Palavras-Chave - Simulação, Equílibrio Geral, Sistemas Econômicos, Dinâmica Estocástica, Linearização. 


\section{ABSTRACT}

The purpose of this academic work is to study a family of models called stochastic economic general equilibrium models (DSGE), and implement a solution to a general model developed model by Smets-Wouters (2003).

We will note in this essay a description of this model, its theoretical foundations, and a simulation of this model, so as to see what the economic policy directions that such a model allows us to understand.

The results obtained using data from the US economy allowed us to infer directions for the variables product and consumption under a innovation shock. Yet we will be able to estimate a direction to the trajectory of PIB for years to next years.

Keywords - Simulation, General Equilibrium, Economic System, Dynamic Stochastic, Linearization. 


\section{LISTA DE FIGURAS}

1 Influência do parâmetro b . . . . . . . . . . . . . . . . . . . 18

2 Influência do parâmetro v . . . . . . . . . . . . . . . . . 18

3 Diagrama de blocos . . . . . . . . . . . . . . . . . . . . . . 19

4 Diagrama de blocos para um modelo de 4 variáveis . . . . . . . . . . . 20

5 Modelo de Crescimento Neoclássico . . . . . . . . . . . . . . . . . 25

6 Crescimento com Retornos Crescentes . . . . . . . . . . . . . . . . 27

7 Crescimento com Retornos Decrescentes . . . . . . . . . . . . . . . . 28

8 Crescimento Ótimo . . . . . . . . . . . . . . . . . 32

9 Modelo de Tempo Contínuo . . . . . . . . . . . . . . . . . . 37

10 Ambiente de funcionamento Dynare . . . . . . . . . . . . . . . . . . . 40

11 Estatística de Akaike . . . . . . . . . . . . . . . . . . . . 48

12 Calibragem do Modelo . . . . . . . . . . . . . . . . . . 50

13 Desvio Padrão da Projeção do Erro das Variáveis Estimadas . . . . . . . . 51

14 Impulso Resposta a um choque . . . . . . . . . . . . . . . . . 51

15 Estimativa de Crescimento do PIB . . . . . . . . . . . . . . 52 


\section{LISTA DE TABELAS}

1 Taxa de Crescimento - Modelo de Solow . . . . . . . . . . . . . . . . 23

2 Base de dados das séries temporais . . . . . . . . . . . . . . . . 43 


\section{SUMÁRIO}

Parte I: INTRODUÇÃO

1 Revisão da Literatura

2 Evolução dos Modelos Macroeconômicos até o DSGE

2.1 O Modelo Neoclássico de Crescimento - Modelo de Solow . . . . . . . . . . 22

2.2 O Modelo Neoclássico de Crescimento - Modelo de Ramsey . . . . . . . . . 23

2.3 O Modelo de Crescimento Ótimo - Modelo de Ramsey-Cass-Koopmans . 28

2.4 O modelo de Equilíbrio Dinâmico Estocástico - DSGE . . . . . . . . . . . 37

2.4.1 Modelo Geral . . . . . . . . . . . . . . . . 37

2.4.2 Equilíbrio Dinâmico do modelo geral . . . . . . . . . . . . . . 39

2.4.3 Solução do modelo geral . . . . . . . . . . . . . . . . . . 40

3 O modelo DSGE de Smets-Wouters $\quad 41$

3.1 Base de Dados . . . . . . . . . . . . . . . . . . . . . . . . . 43

3.2 Ciclos Econômicos . . . . . . . . . . . . . . . . . . . 43

3.3 Especificação do Modelo DSGE . . . . . . . . . . . . . . . . . . . 43

3.4 Ajuste do modelo . . . . . . . . . . . . . . . . . . 47

3.5 Avaliação da Precisão das Projeções do Modelo . . . . . . . . . . . . . . . 49

3.6 Análise Impulso-Resposta . . . . . . . . . . . . . . . . . . . . . 51

3.7 Previsão do PIB . . . . . . . . . . . . . . . . . . . . . . 52

4 Conclusões e Trabalhos Futuros $\quad 53$

Referências $\quad 54$ 


\section{PARTE I}

INTRODUÇÃO 
A gênese deste trabalho está ligada à necessidade de aliar o senso de julgamento intuitivo à uma abordagem econométrica que viesse a permitir o estabelecimento de uma nova estrutura para a formulação de previsões quantitativas das variáveis econômicas como, por exemplo: inflação, crescimento, poupança, etc.

Deste modo nos anos 90 percebeu-se o surgimento de uma classe de modelos denominada de DSGE - dynamic stocasthic general equilibrium -, ou seja, modelos que tratavam os participantes deste modelo em equilíbrio de steady state, e que permitiam em geral abordar o comportamento aleatório dos choques aos quais as variáveis econômicas estão sujeitas.

A teoria do equilíbrio dinâmico aplicada à modelagem de problemas econômicos deu origem aos chamados modelos de equilíbrio dinâmico sujeito a choques estocásticos.

O acrônimo DSGE passou a refletir a incorporação de ferramentas robustas de simulação aos modelos econômicos estruturais, tal novidade permitiu impor uma disciplina de análise sistemática que superou as formas anteriores de trabalho que eram concentradas em um colêtanea de procedimentos ad hoc.

Para os formuladores de política econômica que desejam, por exemplo, informar ao mercado a necessidade de adotar um regime de taxa de câmbio competitiva, ou tratar acerca da sustentabilidade de um déficit comercial, seria mais funcional realizar tais direcionamentos se os mesmos pudessem ser sustentados por uma modelagem econométrica.

Mais do que se valer de gráficos ou cálculos alicerçados em modelos de equilíbrio parcial incompleto, que são baseados em dados do passado corrente, a moderna teoria econômica procura construir modelos macroeconômicos especificados de forma mais completa e que atendam ao requisito de uma lógica interna, e portanto apresentem uma resposta coerente, podendo serem calibrados para capturar as variáveis chave do mundo econômico real.

A forma mais ampla de nos dirigirmos a tal objetivo é por meio da utilização de modelos de equilíbrio geral dinâmico sujeito a choques estocásticos. Os avanços no campo do processamento computacional permitiram que não se resolva estritamente de forma linear os modelos de equilíbrio geral. Até então se fazia necessário linearizar a condição não linear de tais modelos em torno do estado de equilíbrio de longo prazo, steady state, e assim tornar operacionais tais modelos para a estimação, simulação computacional e consequente estimação de política monetária.

O grande argumento a favor da linearização é o de permitir que tanto a estimação quanto a simulação sejam feitas relativamente rápidas. Entretanto, tal tratamento não 
permite que se estude amiúde questões pertinentes a ajustes econômicos, por exemplo, uma resposta assimétrica a choques adversos nos preços dos ativos financeiros. Assim, a depender do objetivo requisitado para estudo, a forma de tratamento da resolução do modelo de equílibrio geral pode oferecer benefícios ou limitações.

Neste trabalho buscamos mapear o problema econométrico em suas variáveis estruturais e descrever tal ambiente como um sistema que pode ser representado por mudanças ao longo do tempo, na medida em que tal sistema passa a ser alimentado por dados entrantes.

Assumimos uma estrutura teórica de modelo mínimo de dinâmica econômica (DSGE), no caso, utilizando o modelo de Smets-Wouters (2003) para uma economia. Apresentamos as previsões à frente para crescimento e as funções impulso resposta para analisar os efeitos dinâmicos dos choques estruturais. 


\title{
1 REVISÃO DA LITERATURA
}

\author{
"Prediction is very difficult, especially if it's \\ about the future" \\ -- Niels Bohr
}

A história do desenvolvimento dos modelos macroeconômicos agregados inicia-se nos anos 80 com a publicação do paper Time to build and Aggregate Fluctuations publicado por Kydland and Prescott (1982), onde pela primeira vez dispunha-se de um modelo dinâmico representativo de uma economia, construído com agentes que buscam otimizar seu bem estar, baseando-se na teoria das expectativas racionais de Lucas (Lucas, 1972) e um mercado eficiente. Tratava-se do primeiro registro de uma publicação onde se procurava explicar o comportamento da variáveis macroeconômicas usando modelos de equilíbrio geral, estocástico, dinâmico (DSGE).

Também nos anos 80 o trabalho de Sims (1980) caracterizava e procurava explicar os movimentos da variáveis econômicas usando Vetor Autoregressivo (VAR).

Estas duas abordagens no tratamento dos problemas macroeconômicos apresentam pontos fortes e pontos de atenção. Os modelos VAR, podem ser estimados diretamente do conjunto de dados, e uma vez estimados, podem ser utilizados para comparações estatítisticas, testes de hipóteses e previsão. Além disso, já que a especificação destes modelos requer pouco detalhamento de teoria econômica, os modelos VAR apresentam um grau de flexibilidade suficiente para endereçar um espectro amplo de questões concernentes à flutuação dos ciclos econômicos. Por outro lado, pelo fato de não necessitarem de um grande arcabouço teórico os modelos VAR podem falhar em tratar parâmetros que são estruturais, assim estes modelos podem exibir instabilidade após mudanças na política monetária, por exemplo. Stock e Watson (1996) encontram evidências de instabilidade nos modelos VAR estimados com dados do pós guerra.

Os modelos DSGE, em contraste, são construídos alicerçados em teoria econômica, ou seja, tais modelos estabelecem conexão entre parâmetros estruturais na medida em que descrevem as preferências dos agentes por consumir, contam com a presença de mudanças tecnológicas, contam também com variáveis econômicas endógenas como produto agregado, nível de emprego. Em princípio, estes parâmetros estruturais deveriam se manter 
invariantes a mudanças de regime de política econômica.

Neste contexto vale abrir um parênteses para comentar a observação de Jesús FernándezVillaverde no working paper The Econometrics of DSGE Models onde ele compara a referida mudança de abordagem que passou a ser fundamentada no tratamento quantitativo via a utilização da teoria do equilíbrio dinâmico à um salto quântico, onde por analogia, em uma geração saltou-se de um protótipo de avião implementado pelos irmãos Wright para um Airbus 380.

Não obstante a simplificação tal modelo foi pensado para compartar os ingredientes necessários que sustentassem a teoria dos ciclos econômicos, quais sejam: moeda, rigidez nominal de preços e mercado não eficiente.

A estrutura básica do modelo apresenta equações que descrevem o comportamento das famílias por consumo, trabalho, produção, custo de decisão e a política monetária.

O modelo na sua forma inicial e, portanto simplificada, não apresenta rigidez 'stickness' na forma de preços ou salários, nem qualquer forma de custo de ajuste. Trata-se, portanto, de um modelo de preços flexíveis e que pode ser entendido como equivalente para uma economia que não apresenta distorções. Não obstante um menor grau de complexidade, ainda assim não se dispõe de uma solução de formato fechado, sendo necessário enveredar pelo caminho da solução por meio de simulação.

Na arquitetura dos modelos DSGE o ambiente econômico representativo na sua representação mais ampla apresenta cinco principais grupos de agentes econômicos. O primeiro grupo é constituído pelas famílias que consomem bens e ofertam trabalho. O segundo grupo é representado pelas firmas que combinam capital e trabalho na produção dos bens que são demandados por domésticos ou por estrangeiros. As firmas tem a capacidade de fixarem preços. Entretanto, inicialmente, consideraremos que os preços são totalmente flexíveis. O terceiro grupo é o da autoridade governamental que configura a política monetária e a política fiscal. O quarto grupo é dos estrangeiros que demandam bens produzidos internamente e oferecem bens produzidos em seus mercados. Por fim, o quinto grupo é o das instituições financeiras que intermediam empréstimos para as famílias.

A representação adotada do modelo econômico é de uma pequena economia aberta, onde a demanda agregada de um país pode ser representada pela seguinte identidade:

$$
D=C+I+G+(X-M),
$$


onde temos o seguinte: as famílias consomem (C) e investem (I), as firmas importam $(\mathrm{M})$, o governo tem o seu gasto corrente com despesas e investimento $(\mathrm{G})$, e para o restante do mundo é exportada demanda (X).

Pelo lado da oferta (Y) estamos num ambiente de firmas competitivas que vendem seus produtos no mercado local e no exterior. As entradas são trabalho, capital e importações.

Antes de abordarmos os modelos DSGE de forma mais aprofundada, é oportuno dar um passo atrás para discorrermos sobre um exemplo econômico de sistema dinâmico discreto linear, o qual pode nos oferecer uma intuição do que este trabalho objetiva alcançar, mas de forma mais aprofundada ao abordar a classe de modelos DSGE.

Para o exemplo simplificado de uma economia fechada que vamos expor, imagine que para um dado país a renda nacional (PIB) é dada no ano $n$ por $P I B_{n}=C_{n}+I_{n}+G_{n}$, onde $C_{n}, I_{n}$ e $G_{n}$ representam, respectivamente, gasto nacional das famílias, investimento privado e gasto do governo. Assumimos neste exemplo que a renda nacional ao crescer de um ano para outro faz com que as famílias também cresçam o seu consumo ao longo destes anos. A premissa utilizada neste exemplo é de que o gasto com consumo em um ano corrente equivale a $\frac{1}{6}$ da renda do ano anterior, ou seja, $C_{n+1}=\frac{1}{6} P I B_{n}$. É esperado que no gasto com consumo também conduza a um aumento nos investimentos privados para o próximo ano, assim, assumimos que $I_{n+1}=C_{n+1}-C_{n}$. Substituindo por $C_{n}$ temos então $I_{n+1}=\frac{1}{6}\left(P I B_{n}-P I B_{n-1}\right)$. Finalmente assumimos que o gasto do governo seja mantido constante. Deste modo, temos o modelo econômico inicial reescrito da seguinte forma,

$$
P I B_{n+2}=\frac{5}{6} P I B_{n+1}-\frac{1}{6} P I B_{n}+G
$$

onde $P I B_{n}$ é a renda nacional no ano $n$, e o gasto do governo $G$ é uma constante. Assumindo que o PIB seja da ordem de grandeza, G e como está crescendo, no ano posterior venha a ser $1,5 G$, ou seja, o PIB expandiu a razão de $0,5 \mathrm{G}$. O nosso interesse com o modelo econômico simplificado que especificamos poderia ser, por exemplo, (a) construir uma solução geral que fornecerá a trajetória da economia, (b) - estimar o PIB após um período de 5 anos, (c) - a convergência de longo prazo da economia. A fim de responder estas perguntas passamos a resolução do modelo econômico simplificado.

A equação característica da equação 1.2 é dada por,

$$
\lambda^{2}-\frac{5}{6} \lambda+\frac{1}{6} \lambda=0
$$


cuja solução é $\lambda_{1}=\frac{1}{2}$ e $\lambda_{2}=\frac{1}{3}$. A equação 1.2 apresenta um termo constante G, logo, assumindo que a solução particular envolve um termo constante teríamos; $P I B_{n}=k_{3} G$, assim a equação 1.2 passaria a ser reescrita nos seguintes termos

$$
k_{3} G=\frac{5}{6} k_{3} G-\frac{1}{6} k_{3} G_{n}+G
$$

e $\operatorname{assim} k_{3}=\frac{1}{1-\frac{5}{6}+\frac{1}{6}}=3$. Portanto, a solução geral assume a seguinte forma

$$
P I B_{n}=k_{1} \lambda_{1}^{n}+k_{2} \lambda_{2}^{n}+3 G
$$

Dando seguimento à utilização do modelo simplificado vamos projetar qual deve ser o PIB esperado para 5 anos à frente, com a seguinte condição $P I B_{0}=G$ e $P I B_{1}=1,5 G$. Isto implica em $k_{1}=-5$ e $k_{2}=3$, quando $n=5$ o PIB $B_{5}=2,856 G$.

Dessa forma, usando 1.5 percebe-se que o comportamento de longo prazo da economia passa a obedecer a seguinte a solução. Na medida em que $n \rightarrow \infty$ temos que $P I B_{n} \rightarrow 3 G$, já que $\left|\lambda_{1}\right|<1$ e $\left|\lambda_{2}\right|<1$. Portanto, a economia estabiliza-se no longo prazo para o valor de $3 G$, ou seja, a solução é estável e convergente sem oscilação, pois $\lambda_{1}$ e $\lambda_{2}$ são ambos menores do que 1 e não apresentam componente imaginário.

Como regra geral, o comportamento da solução pode ser enquadrado em 4 casos possíveis, quais sejam:

- Solução estável, convergente e sem oscilação $\rightarrow$ se $\left|\lambda_{1}\right|<1$ e $\left|\lambda_{2}\right|<1, \lambda_{1}, \lambda_{2}$ reais.

- Solução estável, convergente com oscilação amortecida $\rightarrow$ se $\left|\lambda_{1}\right|<1,\left|\lambda_{2}\right|<1, \lambda_{1}$ ou $\lambda_{2}$ complexos.

- Solução instável e não-oscilatória $\rightarrow$ se $\left|\lambda_{1}\right|>1$ ou $\left|\lambda_{2}\right|>1, \lambda_{1}, \lambda_{2}$ reais.

- Solução instável e oscilatória $\rightarrow$ se $\left|\lambda_{1}\right|>1$ e $\left|\lambda_{2}\right|>1, \lambda_{1}$ ou $\lambda_{2}$ complexos

Ainda nesta linha de raciocínio caso desejássemos entender a sensibilidade do consumo à renda do período anterior como exposto em 1.6, teríamos tal sensibilidade exposta no gráfico abaixo.

$$
C_{t}=a+b Y_{t-1}
$$




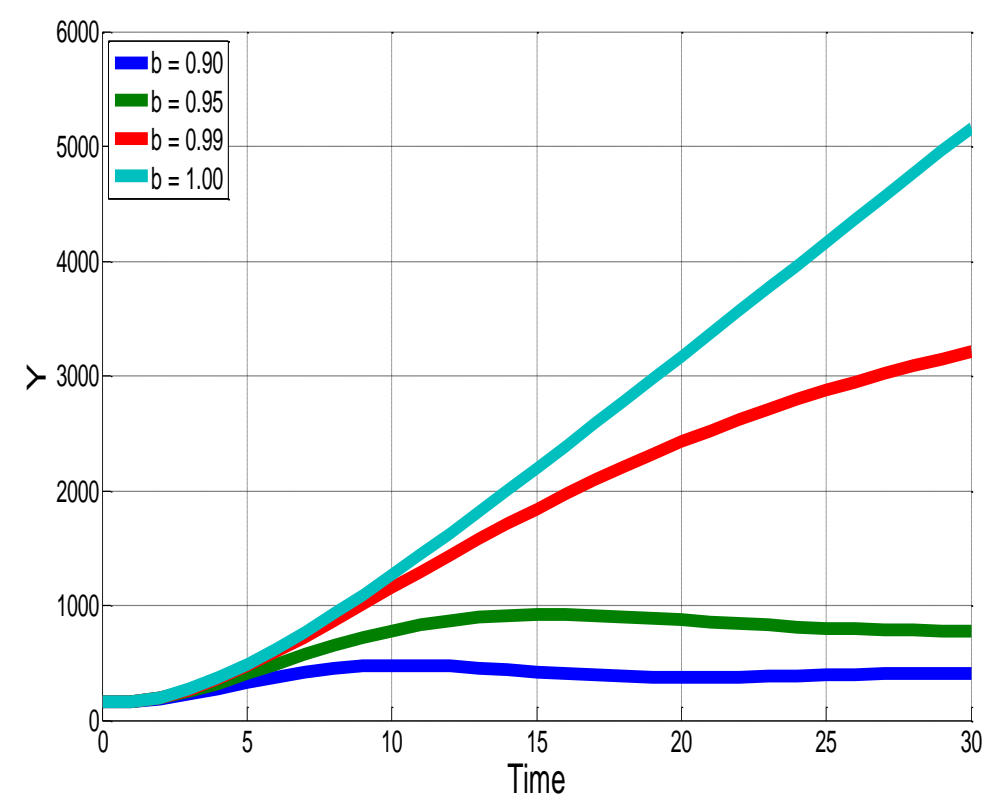

Figura 1: Influência do parâmetro b

Por outro lado, no modelo descritivo da economia vemos que no caso de entendermos o investimento como dependente da renda dos períodos passados, como exposto em 1.6, a sensibilidade passaria a ter o comportamento conforme abaixo.

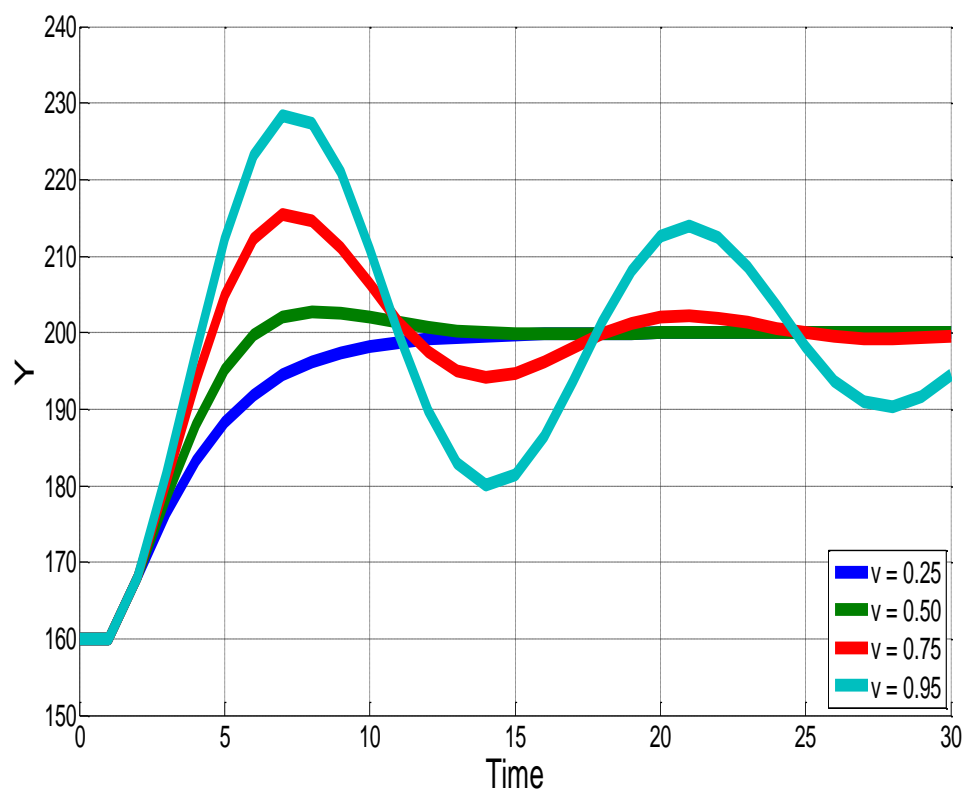

Figura 2: Influência do parâmetro v

O modelo simplificado tem apenas o intuito de oferecer algumas indicações de resultados e de tendência para o ambiente econômico simplificado.

É digno de nota que um enfoque paralelo quanto à modelagem do ambiente econômico 
encontra espaço na teoria de controle ótimo, ou seja, segundo tal arcabouço teórico passase a analisar o sistema econômico como um sistema a ser controlado. Neste caso, o objetivo é fazer com que o erro seja o menor possível. O trabalho de Chow, G. C. (1975), Analysis and Control of Dynamic Systems, é bem conhecido no sentido de apresentar tal abordagem.

$\mathrm{Na}$ teoria de controle a chave para estas questões são (a) como descrever o sistema a ser controlado, ou seja, a modelagem strictu sensu, e, (b) como projetar o controlador, melhor dizendo, o controle. A figura 3 mostra o bloco do esquema geral de controle.

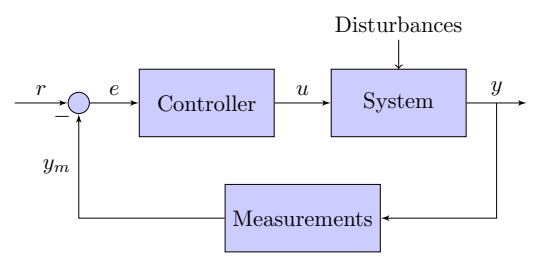

Figura 3: Diagrama de blocos

A seguir apresentamos um esquema de diagrama de blocos, que é a representação gráfica de um modelo de um sistema complexo, no qual tem-se 4 variáveis representativa da modelagem básica de um sistema econômico. Poderá ser notado o fluxo entre variáveis e blocos. Quanto ao mecanismo de feedback, estes são essencialmente fluxos circulares, e o exemplo na figura 4 permite notar o loop entre consumo e PIB (renda nacional). Isto posto, vê-se que consumo é em certa medida determinado pelo PIB, mas é também um componente do PIB.

A análise via diagrama de blocos permite que se identifique no modelo os mecanismos que poderiam resultar em desempenho instável. No exemplo apresentado, tanto o consumo como o investimento, dependem do PIB, ao passo que ao mesmo tempo contribuem para a formação do PIB. Logo, se o multiplicador de coeficientes nestas equações é muito grande, um loop feedback poderia implicar que, de forma continuada, pequenas variações no consumo ou na demanda por investimento resultassem em grandes variações de PIB. A política de controle que poderia ser derivada é uma reestruturação das equações de demanda de forma a reduzir a dependência do PIB. Seria possível também supor a introdução de uma outra variável explicativa que poderia eliminar a forte dependência do PIB. Ao se tratar de reestruturação de modelo, uma preocupação que deve ser lembrada é que, diferentemente de modelos de equações simples, onde a busca é por uma melhor estatística de fit do modelo, no caso de modelos multi-equações a preocupação é com interação dinâmica das equações que compõem o modelo. Sendo possível existir uma situação onde a reestruturação das equações fosse levada a frente, não obstante um nível 


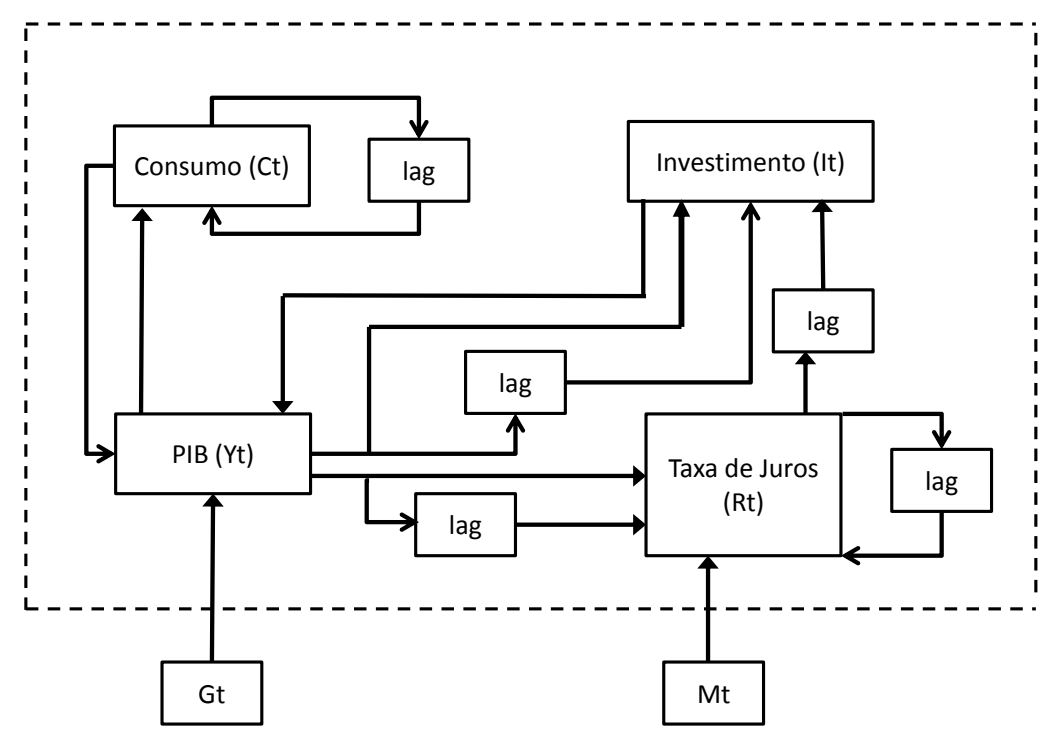

Figura 4: Diagrama de blocos para um modelo de 4 variáveis

razoávelmente bom das estatísticas de fit das equações.

Retomando a abordagem da família de modelos DSGE, tem-se que se considera a rigidez de preços e salários segundo a regra de Calvo, ou seja, a cada período uma fração das famílias pode corrigir os seus salários, e as demais famílias podem apenas parcialmente indexar seus salários pela inflação passada.

O governo nos modelos DSGE segue uma regra de política monetária, conforme especificação particular.

Para o mundo exterior as variáveis de interesse a serem consideradas são: taxa de juros, inflação, importação do resto do mundo e aversão a risco por parte dos investidores externos.

O termo estocástico surge no modelo por meio de choques que podem ser das seguintes naturezas: choques de investimento, choques de política monetáaria, choques de política fiscal, etc. Ainda no que concerne aos choques nas suas diversas modalidades surge o fator persistência, que é responsável por representar o efeito extendido da duração da resposta da economia a um choque. O fator persistência pode ser entendido como uma persistência de hábito de consumo, ou, um ajustamento a um custo de investimento, ou, por fim, a uma mudança da taxa de utilização de capital.

A abordagem da idéia básica dos modelos de equilíbrio geral consiste na interação das famílias que procuram maximizar a sua função utilidade e das firmas que buscam maxi- 
mizar a sua função lucro num mundo com recursos finitos. A metáfora a este modelo de equilibrio geral é o pensamento de um planejador central que tem por objetivo determinar o ponto ótimo de maximização de utilidade das famílias (ótimo de Pareto).

Recentemente o trabalho de Bernanke (1986), Blanchard e Watson (1986), e Sims (1986), procurou mostrar a associação do poder da teoria econômica com a flexibilidade dos modelos VAR.

Por fim, alguns pesquisadores tem adotado a linha de impor restrições que julguem ser plausíveis dentro da teoria econômica aos seus modelos VAR, tal linha de pesquisa é conhecida por 'structural VAR'. Esta restrição se mostra na matriz A, conforme veremos mais adiante. McKibbin et al. (1998) tem desenvolvido trabalhos neste sentido. 


\section{EVOLUÇÃO DOS MODELOS MACROECONÔMICOS ATÉ O DSGE}

\subsection{O Modelo Neoclássico de Crescimento - Modelo de Solow}

A questão fundamental de longo prazo para a Macroeconomia gira em torno do caminho "time path"' da economia. Neste sentido perguntas como (i) - O que determina o caminho/trajetória da economia?, (ii) - Como o caminho/trajetória pode ser alterado?, (iii) - Se um economia está distante do caminho/trajetória será possível imaginar o retorno ou jamais haverá um retorno?

As perguntas acima passaram a ter a sua resolução endereçada pelo economista Roberto Solow. Em sua modelagem macroeconômica Solow procura explicar como as variáveis produto $(\mathrm{Y})$, capital $(\mathrm{k})$ e trabalho (L) ajustam-se no tempo, ou seja, a dinâmica é o ponto central da análise.

O modelo de Solow assume que Y, K, L e a tecnologia são fixos. Contudo permanece relevante o entendimento de como o produto é alocado pois a alocação determina alterações no produto futuro.

Tomemos um breve exemplo para retratar o exposto até o momento. Considere duas economias, A e B. Na economia A todo o produto é consumido; enquanto na economia B metade do produto é destinado ao investimento. O que seria esperado imaginar acerca do desempenho dessas duas economias, A e B, ao longo do tempo? Parece óbvio supor que a economia A permanecerá estagnada, ou até decairá por conta da depreciação, enquanto B crescerá? Afinal, a economia B está investindo, ou seja, adicionando novas fábricas e equipamentos, que podem ser utilizados para crescer o produto para o próximo ano.

O principal foco do modelo de Solow é em como o produto é alocado em investimento e como tal relação varia ao longo do tempo. Em suma, como o caminho/trajetória das variáveis é objeto de estudo dizemos que trata-se de modelo de dinâmica macroeconômica. 
A seguir apresentamos um exemplo ilustrativo bem simples, mas ilustrativo do conceito que subjacente ao modelo de crescimento de Solow.

Suponha que no tempo inicial disponhamos de 100 unidades de capital e de trabalho, e estes fatores de produção gerem 100 unidades de produto no ano 1, pois capital e trabalho operam na razão de meio para a formação do produto. Com o resultado obtido das 100 unidades de produto dividimos metade em consumo e investimento. No passo seguinte, ao adicionarmos 50 unidades de capital ao estoque de 100 unidades de capital totalizamos 150 unidades de capital. Utilizando capital e trabalho no ano 2 teremos um produto de 122,48 . Mantendo-se a mesma razão teremos no ano 3 um produto de 145,34 e no ano 4 um produto de 168,49 .

$\begin{array}{ccccc} & \text { Ano 1 } & \text { Ano 2 } & \text { Ano 3 } & \text { Ano 4 } \\ \text { Capital } & 100 & 150 & 211 & 284 \\ \text { Trabalho } & 100 & 100 & 100 & 100 \\ & & & & \\ \text { Produto } & 100 & 122,5 & 145,3 & 168,5 \\ \text { Taxa de Crescimento } & 22,5 \% & 18,7 \% & 15,9 \% \\ \text { 1/2 Consumo } & 50 & 61,24 & 72,67 & 84,25 \\ \text { 1/2 Investimento } & 50 & 61,24 & 72,67 & 84,25 \\ \text { Tabela 1: Taxa de Crescimento - Modelo de Solow }\end{array}$

Deste pequeno exercício podemos observar que a economia cresceu ao longo do tempo com acumulação de capital, o que é a idéia básica do modelo de Solow. Não obstante tal constatação o fato que podemos apontar é que a taxa de crescimento é decrescente, ou seja, a acumulação de capital per se não é suficente para explicar um crescimento continuado da economia.

\subsection{O Modelo Neoclássico de Crescimento - Modelo de Ramsey}

Na história dos modelos econométricos que procuram descrever o funcionamento de uma economia surge o modelo de Frank P. Ramsey, "A Mathematical Theory of Saving" Economic Journal, December 1928, pp.543-559, que exerceu uma grande influência no pensamento econômico após a Segunda Guerra Mundial, tornando-se massa crítica de discussão pelos economistas teóricos estudiosos das teorias de crescimento econômico.

O modelo de Ramsey lançou um olhar detalhado sobre uma questão de relevante importância que é a alocação intertemporal de recursos. Dito de outro modo, a questão 
a ser respondida é quanto da riqueza nacional (produto nacional) produzida num dado instante de tempo seria destinada para o consumo corrente a fim de produzir utilidade corrente, e quanto seria poupada ou investida, de modo a expandir a produção e consumos futuros, e consequentemente gerar utilidade futura para as famílias.

Em papers desenvolvidos recentemente a tese central é formulada como um problema que encaixa-se na teoria de controle ótimo cuja função objetivo é a utilidade. Além disso, o novo tratamento rotulado como teoria neoclássica de crescimento ótimo, estendeu o modelo de Ramsey em duas frentes principais:

- Assume-se que a força de trabalho (população) cresce a uma taxa exógena constante $n>0$ (no modelo de Ramsey $n=0$ );

- A utilidade passa a ser tratada sob a forma de um desconto temporal a uma taxa constante $\rho>0$ (no modelo de Ramsey $\rho=0$ ).

O modelo é enquadrado como pertencente a teoria 'neoclássica' porque sua estrutura analítica gira em torno da função de produção neoclássica $\mathrm{F}=\mathrm{F}(\mathrm{K} ; \mathrm{L})$ onde assume-se retornos constante de escala, onde L é o trabalho e K o estoque de capital. Quando o retorno do capital é isento de taxação 'tax free' a teoria equivale a taxa bruta de retorno, $R_{t+1}$, ao produto marginal bruto do capital descontada a taxa de depreciação, ou seja, $F_{k, t+1}+(1-\delta)$, onde $F_{k, t+1}$ é o produto marginal do capital e $\delta$ é a taxa de depreciação.

O modelo neoclássico de crescimento originado com o trabalho de Solow e Swan consiste da seguinte relação de variáveis: uma função de produção $y_{t}=f\left(h_{t}, k_{t}\right)$, onde $y_{t}$ representa o produto, $h_{t}$ é o trabalho, e $k_{t}$ é o capital no momento $\mathrm{t}=0,1,2, \ldots$

Adicionalmente temos uma lei de movimentação do estoque de capital, $k_{t+1}=(1-$ $\delta) k_{t}+\sigma \cdot y_{t}$, onde $\delta \in(0,1)$ é a taxa de depreciação e $\sigma \in(0,1)$ é a taxa de poupança.

Assume-se que $f$ é homogênea com grau de liberdade 1, crescente, côncava e duas vezes diferenciável continuamente, também é assumido que $h_{t}$ é constante e normalizada $h_{t}=1$. Pode-se argumentar que tal hipótese não é muito próxima da realidade observada, entretanto, nos próximos passos ao se endogenizar $h_{t}$ os agentes representativos da economia, que se deseja retratar, definirão o tempo a ser destinado para trabalho, e a quantidade de tempo a ser destinada para outras atividades, por exemplo, o lazer.

Seja $F_{k}=f(1, k)$, então $F_{k}^{\prime}>0, F_{k}^{\prime \prime}<0$. Adicionalmente, assume-se que $F(0)=0$, $F^{\prime}(0)=\infty$ e $F^{\prime}(\infty)=(0)$, e o estoque de capital inicial, $k_{0}$, é dado exogenamente. 
Note que impõem-se exogenamente o seguinte comportamento para um indivíduo representativo do sistema econômico a ser modelado, - o número fixo de horas trabalhadas é $h_{t}=1$, a destinação para a poupança ou investimento é dada por $i_{t}=\sigma y_{t}$, e o consumo é representado por $c_{t}=(1-\sigma) y_{t}$ em cada período de tempo. Substituindo a função produção na lei de movimento de capital obtém-se a equação de diferenças de primeira ordem em $k_{t}$.

$$
k_{t+1}=(1-\delta) k_{t}+\sigma F\left(k_{t}\right) \equiv g\left(k_{t}\right)
$$

Agregando-se a condição inicial $k_{0}$, a equação acima determina inteiramente o caminho temporal do estoque de capital. Dado tal caminho determina-se os caminhos para $y_{t}, c_{t}$, etc e um steady state do sistema é uma solução para $k=g(k)$.

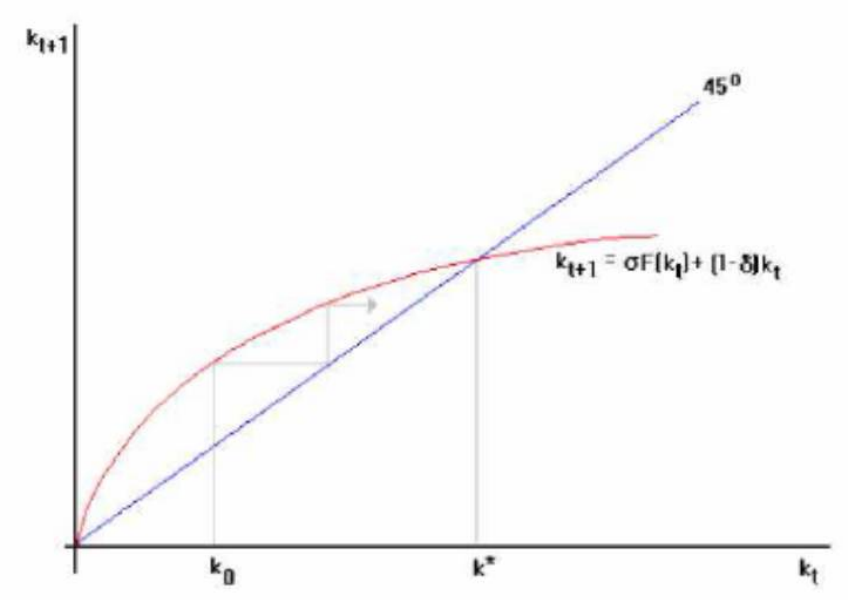

Figura 5: Modelo de Crescimento Neoclássico

O gráfico acima representa $g(k)$ versus $\mathrm{k}$, vale ressaltar que as hipóteses assumidas implicam que $g(0)=0, g^{\prime}(0)>1$, e há um único $k^{*}>0$ tal que $k^{*}=g\left(k^{*}\right)$.

Consequentemente, o modelo representa dois steady states, $k=0$ e $k=k^{*}$, e para todo $K_{0}>0, K_{t} \rightarrow k^{*}$ (monotonicamente).

Consequentemente como $t \rightarrow \infty, y \rightarrow y^{*}, c_{t} \rightarrow c^{*}$, etc. Em $k^{*}$ temos $\left(k^{*}\right)=\delta k^{*}$, que implica que a poupança apenas compensa a depreciação e a razão capital-produto é dada por $\frac{k}{y}=\frac{\sigma}{\delta}$, e também que $c^{*}=y^{*}-\delta k^{*}$. Claramente $K^{*}$ é crescente em $\frac{\delta}{\sigma}$. Além disso, $c^{*}$ é primeiramente crescente, e então descrescente em $\delta$. A taxa de poupança que maximiza 
o consumo em steady state pode ser mostrada que satisfaz $F^{\prime}\left(K^{*}\right)=\sigma$ que é chamada de regra de ouro de Phelps de acummulação de capital.

Observe que o modelo básico de crescimento não apresenta efetivamente um crescimento de longo prazo, ou seja, para todo $K_{0}>0$, com $t$ crescente $k_{t}$ converge para $k^{*}$.

Assintoticamente, o crescimento cessa, e isto deve-se a hipótese que o produto marginal do capital torna-se pequeno na medida em que o estoque de capital aumenta, melhor dizendo, trata-se da hipótese que $F^{\prime}(k) \rightarrow 0$ com $k \rightarrow \infty$. Suponha que relaxemos esta hipótese, ou seja, passemos a assumir uma condição de tecnologia linear, $F(k)=A k$ para qualquer constante $A>0$.

Então o caminho temporal de $k_{t}$ satisfará:

$$
k_{t+1}=(1-\delta) k_{t}+\sigma A k_{t}=B K_{t}
$$

onde $B \equiv 1-\delta+\sigma A$.

Isto implica que $K_{t}=B^{t} k_{0}$ para todo $t$. Se $B>1$ então a economia em questão cresce sempre à taxa de $B-1=\sigma A-\delta$, enquanto que se $B<1$ então $K_{t} \rightarrow 0$. Em qualquer caso, produto, consumo e investimento crescem todos à mesma taxa de crescimento, $k_{t}$. Conclue-se que para termos taxa de crescimento de longo prazo, em tal configuração, a taxa de poupança $\sigma$ e a produtividade $A$ devem ser altas relativamente à depreciação $\delta$.

É possível ir além da inserção da tecnologia linear e assumir que $F(k)$ exibe retornos crescentes, ou seja, $F^{\prime \prime}>0$. Supondo que $g^{\prime}(0)<1$, que é verdadeiro somente e somente se, $F^{\prime}(0)<1-\delta \sigma$, como mostrado na figura a seguir. Então existe um único steady state $k *$ que é instável para $k_{0}<k^{*}$ e para $K_{0}>k *, k_{t}$ apresenta crescimento explosivo.

Se $F(k)$ exibe primeiramente retornos crescentes e depois então retornos decrescentes, então como mostrado na figura a seguir existem dois steady states, um estável e um instável. Estes dois exemplos mostram como um pequeno estoque de capital pode ser uma condenação para uma economia conduzindo-a para uma baixa taxa de crescimento ou um baixo nível de produto. Além disso, pequenas mudanças na condição inicial podem fazer uma grande diferença para o comportamento assintótico da economia.

Uma forma alternativa para gerar crescimento persistente é retornar para um padrão tecnológico côncavo e adicionar um progresso tecnológico exógeno. Por exemplo, suponha que $y_{t}=f\left(z_{t} h_{t}, k_{t}\right)$ onde $z^{t}=\gamma^{t}$ representa uma mudança tecnológica determinística. 


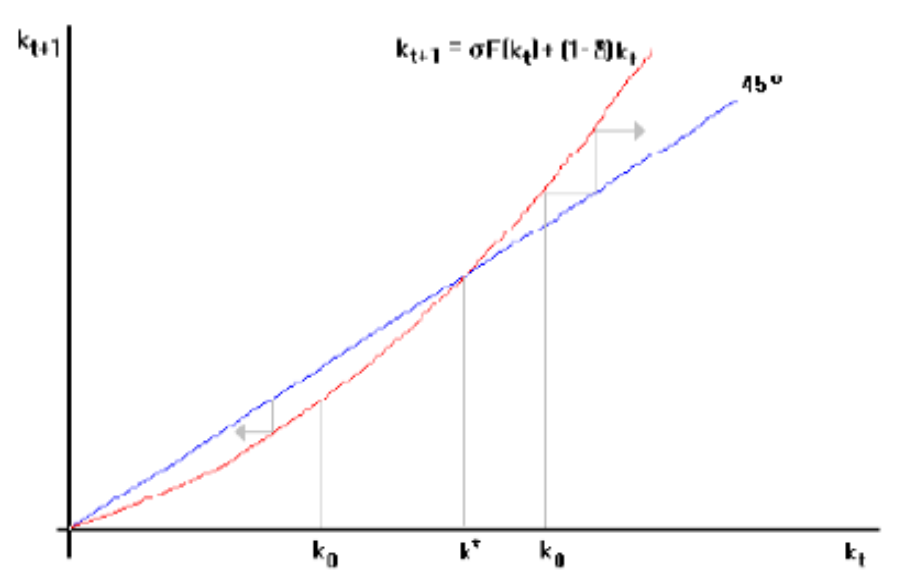

Figura 6: Crescimento com Retornos Crescentes

Note que a mudança tecnológica aqui é responsável por aumentar o trabalho, isto é necessário para obtermos um caminho balanceado de crescimento no qual capital e produto crescem na mesma taxa quando o trabalho é fixo para qualquer função de produção diferente de uma Cobb-Douglas. No caso Cobb-Douglas pode-se interpretar mudanças tecnológicas como trabalho ou capital, expansoras ou neutras, desde que o termo $z_{t}$ possa ser fatorado.

Então:

$$
k_{t+1}=(1-\delta) k_{t}+\sigma f\left(\gamma^{t}, k_{t}\right)
$$

Seja $\tilde{k}_{t}=k_{t} \gamma^{-t}$. Então a expressão anterior é equivalente a

$$
\gamma^{t+1} \tilde{k}_{t+1}=(1-\delta) \gamma^{t} \tilde{k}_{t}+\sigma f\left(\gamma^{t}, \gamma^{t} \tilde{k}_{t}\right)
$$

Dividindo-se por $\gamma^{t+1}$ e usando a homogeneidade de $f$ pode-se reduzir como segue abaixo:

$$
\tilde{k}_{t+1}=1-\tilde{\delta} \tilde{k}_{t}+\tilde{\sigma} f\left(1, \tilde{k}_{t}\right)=g\left(\tilde{k}_{t}\right)
$$

onde $1-\tilde{\delta}=\frac{1-\delta}{\gamma}$ e $\tilde{\delta}=\frac{\delta}{\gamma}$. Consequentemente, após uma transformação de variáveis 


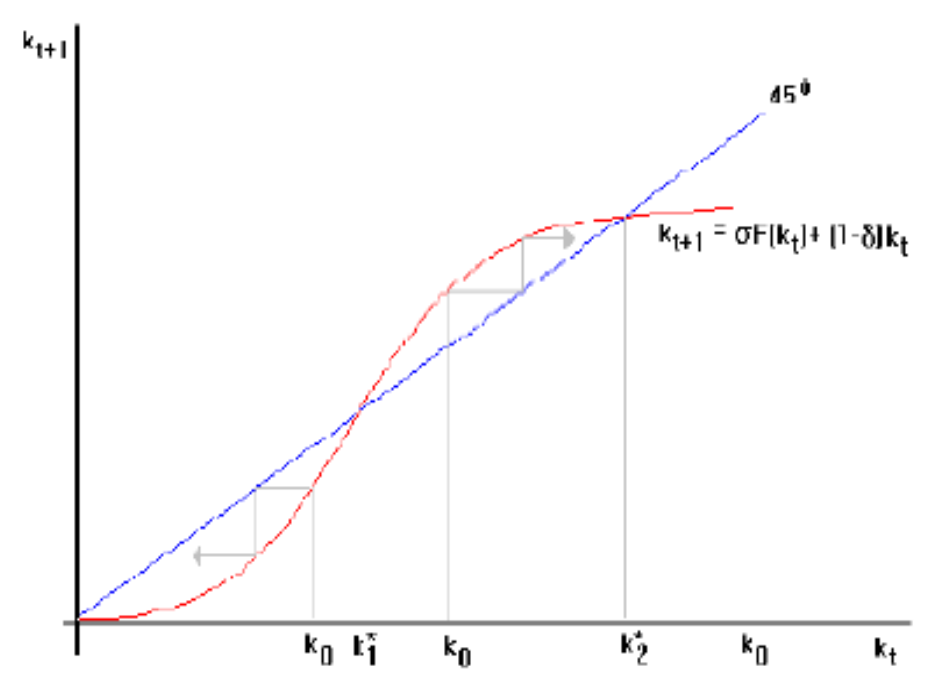

Figura 7: Crescimento com Retornos Decrescentes

o modelo com tecnológica exógena parece exatamente como o modelo de crescimento padrão. Para qualquer $\tilde{k}_{0}>0, \tilde{k}_{t} \rightarrow \tilde{k}^{*} \operatorname{com} t \rightarrow \infty$, onde

$$
\frac{\tilde{k}^{*}}{F\left(\tilde{k}^{*}\right)}=\frac{\tilde{\sigma}}{\tilde{\delta}}=\frac{\sigma}{\delta+\gamma-1}
$$

Invertendo nossa transformação, $\tilde{k}_{t}=\tilde{k}^{*}$ transforma em $k_{t}=\gamma^{t} \tilde{k}^{*}$. Isto significa que assintoticamente $k_{t}$ cresce a mesma taxa do aumento de trabalho num contexto de progresso tecnológico. Certamente produto, e portanto consumo e investimento, asintoticamente também crescem a mesma taxa. Isto produz, referenciando-se em um caminho de crescimento balanceado, que é do mesmo tipo de comportamento apresentado pelo modelo com $y=A k$, e é uma aproximação razoável e consistente com o comportamento apresentado pelas economias desenvolvidas atuais.

\subsection{O Modelo de Crescimento Ótimo - Modelo de Ramsey-Cass-Koopmans}

No modelo de crescimento descrito até então não há decisão econômica a ser tomada: os indíviduos (famílias) simplesmente poupam uma fração constante $\sigma$ do produto. Alternativamente, Cass Koopmans assumem que os indivíduos tem preferências em relação ao consumo, e pensam acerca do modo de como tomar decisões de uma forma objetiva. 
Sendo um pouco mais preciso, imaginemos que todos os consumidores tenham preferências idênticas e que suas decisões sejam coordenadas por um planejador social.

Consideraremos a seguir a relação entre o planejador social e o equilíbrio competitivo descentralizado. O problema do planejador é o que segue:

$$
\max V=\sum_{t=0}^{\infty} \beta^{t} U\left(C_{t}\right)
$$

sujeito a

$$
k_{t+1}=F\left(k_{t}\right)+(1-\delta) k_{t}-c_{t}
$$

e, dado o estoque de capital inicial, $k_{0}$. Impõem-se que $U^{\prime}>0, U^{\prime \prime}<0$ e impõem-se também todas as premissas do modelo básico de crescimento neoclássico sobre $F$. Se substituirmos a restrição dentro da função objetivo, o problema do planejador central é dado por:

$$
\max V=\sum_{t=0}^{\infty} \beta^{t} U\left(F\left(k_{t}\right)+(1+\delta) k_{t}-k_{t+1}\right)
$$

O elemento de escolha é a sequência de capital inicial $k_{t}$ com valor inicial dado $k_{0}$. O problema recursivo descrito acima enquadra-se bem dentro de uma estrutura de programação dinâmica. Vamos tomar a variável de estado como sendo $k_{t}$, e as variável de controle $k_{t+1}$. A lei de movimento é simplesmente o valor do próximo período da variável de estado que é dada pelo valor no período atual da variável de controle ${ }^{1}$. A equação de Bellman é apresentada a seguir.

$$
V\left(K_{t}\right)=\max _{k_{t+1}}\left\{U\left[F\left(k_{t}\right)+(1-\delta) k_{t}-k_{t+1}\right]+\beta V\left(k_{t+1}\right)\right\}
$$

Hipóteses padronizadas e já discutidas na literatura acadêmica garantem que existe unicidade de solução de $V$ na expressão 2.10 para um espaço delimitado de funções contínuas. Pode ser mostrado que $V$ é diferenciável até segunda ordem, estritamente crescente e estritamente côncava sob hipóteses padrão para $f$ e $U .^{2}$

Assumiremos que a solução do problema de maximização do lado direito da equação

\footnotetext{
${ }^{1}$ Como alternativa a variável de controle pode ser também consumo $c_{t}$, ou investimento, $i_{t}$, por conta da identidade: $k_{t+1}=F\left(k_{t}\right)+(1-\delta) k_{t}-c_{t}=(1-\delta) k_{t}-i_{t}$

${ }^{2}$ Detalhamento encontra-se em Stokey 1989 e Santos 1991
} 
de Bellman é interior, ou seja, $0<K_{t+1}<F\left(k_{t}\right)+(1-\delta) k_{t}$, isto pode ser assumido sobre hipótese padrão para $f$ e $U$.

Em seguida, a escolha maximizada de $k_{t+1}$ é caracterizada pela condição de primeira ordem.

$$
U^{\prime}\left(F\left(k_{t}\right)+(1-\delta) k_{t}-k_{t+1}\right)=\beta V^{\prime}\left(k_{t+1}\right)
$$

Seja $k_{t+1}=\alpha\left(k_{t}\right)$ a solução para a condição de primeira ordem como função de $k_{t}$ que é única porque $V$ é estritamente côncava. Dito de outro modo, a função $\alpha(k)$ é definida por:

$$
U^{\prime}\left(F\left(k_{t}\right)+(1-\delta) k_{t}-\alpha k\right)=\beta V^{\prime}\left(k_{t}\right)
$$

A política ótima para este problema de programação dinâmica é dada pela regra de decisão invariante no tempo $k_{t+1}=\alpha(k)$. Tal regra de decisão é uma equação de diferenças de primeira ordem que juntamente com a condição inicial, $k_{0}$, caracteriza completamente a sequência ótima de estoque de capital, $k_{t}$. Uma inferência inicial é a de que $\alpha(0)=0$. Pelo teorema da função implícita sabemos que $\alpha(k)$ é diferenciável. Diferenciando e usando o resultado que $V$ é duas vezes diferenciável e côncava temos:

$$
\alpha^{\prime}(k)=\frac{\left[F^{\prime}(k)+1-\delta\right] U^{\prime \prime}(c)}{U^{\prime \prime}(c)+\beta V^{\prime \prime} \alpha[k]}>0
$$

Consequentemente, $\alpha$ é estritamente crescente. Poderia-se derivar a solução de um modo alternativo, neste caso a idéia é eliminar $V^{\prime}\left(k_{t+1}\right)$ da equação 2.11. A fim de atingir este objetivo derivamos 2.10 .

$$
V^{\prime}\left(k_{t}\right)=U^{\prime}\left[c\left(k_{t}, k_{t+1}\right)\right]\left[F^{\prime}\left(k_{t}\right)+[1-\delta]\right]
$$

Inserindo $c_{t}=c\left(k_{t} ; k_{t+1}\right)=F\left(k_{t}\right)+(1-\delta) k_{t}-k_{t+1}$. Dado que 2.14 é válida para qualquer data $(t)$ pode-se atualizar os subscritos de indexação temporal de $t$ para $t+1$, e substituindo o resultado na equação 2.11 obtemos a chamada Equação de Euler.

$$
U^{\prime}\left[c\left(k_{t}, k_{t+1}\right)\right]=\beta U^{\prime}\left[c\left(k_{t+1}, k_{t+2}\right)\right]\left[F^{\prime}\left(k_{t+1}\right)+1-\delta\right]
$$

Consequentemente, a taxa marginal de substituição entre o consumo em $t$ e em $t+1$ 
dada por $\mu=\frac{u^{\prime}\left(c_{t}\right)}{\beta u^{\prime}\left(c_{t+1}\right)}$ iguala a taxa marginal de transformação, que é dada por $F^{\prime}\left(k_{t+1}\right)+$ $1-\delta$, sendo que isto representa quanto de produto adicinal é obtido no próximo período a partir de uma unidade adicional de poupança.

Note que 2.15 é uma equação de diferenças de segunda ordem em $k_{t}$. Uma sequência ótima $\left\{k_{t}\right\}$ deve satisfazer a equação de Euler, mas desde que seja uma equação de segunda ordem com somente uma condição inicial, $k_{0}$. Existem muitas sequências $\left\{k_{t}\right\}$ que satisfazem 2.15, mas nem todas são ótimas, portanto, tem-se que a equação de Euler é uma condição necessária. Pode-se demonstrar que a sequência $\left\{k_{t}\right\}$ que satisfaz 2.15 é ótima e atende a condição de transversalidade.

$$
\lim _{t \rightarrow \infty} \beta^{t} U^{\prime}\left(c_{t}\right)\left[F^{\prime}\left(k_{t}\right)+1-\delta\right] k_{t}=0
$$

Alternativamente utilizando 2.15 para eliminar $\left[F^{\prime}\left(k_{t}\right)+1-\delta\right]$, pode-se escrever 2.16 como sendo,

$$
\lim _{t \rightarrow \infty} \beta^{t-1} U^{\prime}\left(c_{t-1}\right) k_{t}=0
$$

De modo a entender a condição de transversalidade é muito útil interpretar o problema do planejador central como sendo o limite de sequências de problemas de horizonte finito. Deste modo, considere a escolha de $\left\{k_{t}, \ldots, k_{t+1}\right\}$ para resolver o problema abaixo.

$$
\max V=\sum_{t=0}^{T} \beta^{t} U\left(F\left(k_{t}\right)+(1-\delta) k_{t}-k_{t+1}\right)
$$

O desenvolvimento do problema de controle ótimo impõem as condições de KuhnTucker, ou seja,

$$
\frac{\partial V}{\partial K_{t}}=-\beta^{t-1} U^{\prime}\left(c_{t-1}\right)+\beta^{t} U^{\prime}\left(c_{t}\right)\left[F^{\prime}\left(k_{t}\right)+1-\delta\right] \leq 0, \text { se } k_{t}>0 \text { para } t=1, \ldots, T, \mathrm{e}
$$
no último período, $\frac{\partial V}{\partial K_{T+1}}=-\beta^{T} U^{\prime}\left(C_{T}\right) \leq 0$, se $K_{T+1}>0$.

Hipóteses podem ser feitas para garantir que $K_{t}>0$ de modo que as condições de Kuhn-Tucker mantenham a igualdade para $t=1, \ldots, T$ que produzem a equação de Euler.

Mas enquanto $U^{\prime}\left(c_{t}\right)>0$ não teremos $K_{T+1}>0$. Se escrevermos as condições de Kuhn-Tucker para o último período como $K_{T+1} \frac{\partial V}{\partial K_{T+1}}=K_{T+1} \beta^{T} U^{\prime}\left(c_{t}\right)=0$, então tomando o limite de $T \rightarrow \infty$ produziremos 2.17. Como decorrência, a equação de Euler e a condição de transversalidade caracaterizam o problema do horizonte infinito. 
A condição inicial $k_{0}$ juntamente com a regra de decisão $k_{t+1}=\alpha\left(k_{t}\right)$ determina completamente o espaço $k_{t}$. No ponto de steady state temos que capital, produto, consumo e investimento são todos invariantes no tempo. Pode-se notar na equação 2.15 que o estoque de capital em steady state é tanto $k=0$ ou $k=k^{*}$ onde $k^{*}$ satisfaz $F^{\prime}\left(k^{*}\right)=$ $\delta-1+\frac{1}{\beta}$. A curvatura apresentada de $\mathrm{F}$ assegura que existe um único $K^{*}$. Deixando a taxa de preferência intertemporal $\delta$ ser definida por $\beta=\frac{1}{(1+\delta)}$, pode-se escrever a condição de steady state compactamente como $F^{\prime}\left(k^{*}\right)=\delta+\rho$. Pode-se notar que o steady state do problema de crescimento ótimo difere da solução do problema de maximizar o steady state do consumo que foi dado pela "regra de ouro", $F^{\prime}\left(k^{*}\right)=\delta$, na medida em que $\rho>0$.

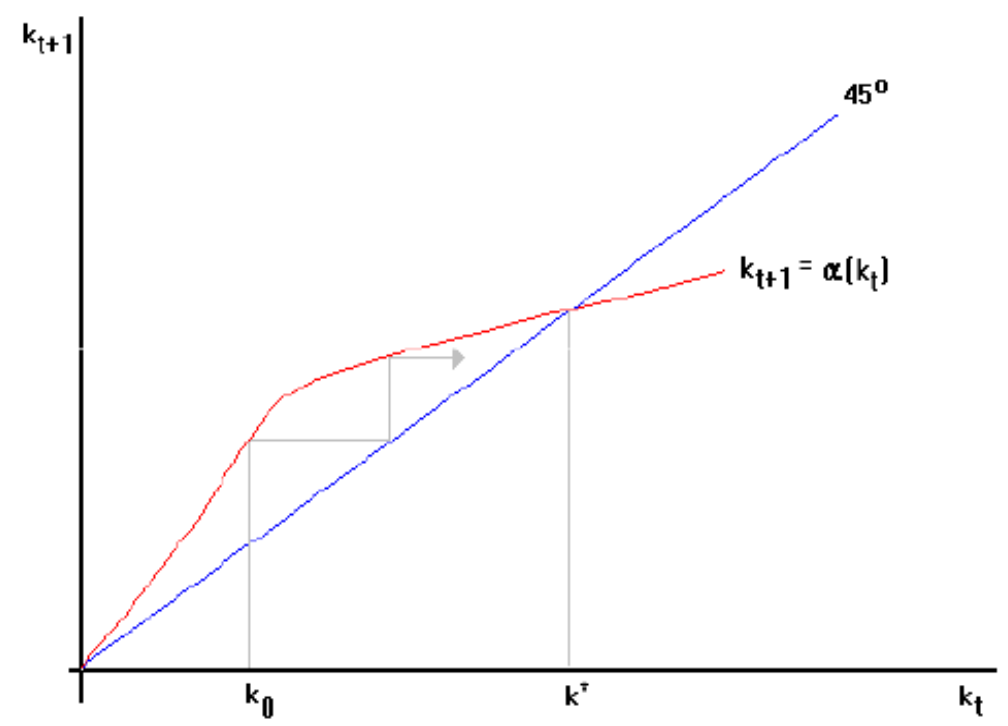

Figura 8: Crescimento Ótimo

Passemos agora a considerar um comportamento distante do steady state. É possível mostrar que a inclinação da regra de decisão em steady state é menor do que 1. Isto posto, como mostrado na 8, $\alpha_{k}$ corta a linha de 45 graus acima em $k^{*}$. Isto significa que para qualquer $K_{0}>0, K_{t}$ converge monotonicamente para $k^{*}$ quando $t \rightarrow \infty$.

Abordando qualitativamente o comportamento de $k_{t}$ temos o mesmo comportamento exposto no modelo de crescimento neoclássico com taxa de poupança fixa $\sigma_{t}$ (vide gráficos 5 e 8). Contudo é importante derivar analiticamente a regra de decisão que governa $\sigma_{k}$ pela seguinte motivação: Suponha ser importante determinar os efeitos de uma mudança marginal em uma variável exógena qualquer e sua respectiva influência sobre $\sigma$. Deste modo, a seguir apresentaremos um exemplo onde é possível analiticamente derivar a solução. No texto de Long \& Plosser (1983) assume-se $U(c)=\log (c), F(k)=K^{\theta}$, e $\delta=1$. 
Obtemos a política ótima e a função valor por meio da iteração da equação de Bellman. Como primeiro passo impõem-se $V_{0}\{k\}=0$, e escrevemos.

$$
V_{1}\left(k_{t}\right)=\max _{k_{t+1}}\left\{\log \left(k_{t}^{\theta}-k_{t+1}\right)+0\right\}
$$

A equação acima pode ser interpretada como a equação de Bellman para o problema do horizonte finito na data final. A solução para o problema de maximização é a regra de decisão $k_{t+1}=\alpha_{1}\left(k_{t}\right)=0$, já que ninguém poupa na data final, isto implica que,

$$
V_{1}\left(k_{t}\right)=\log \left(k_{t}^{\theta}\right)=\theta \log \left(k_{t}\right)
$$

Para o segundo passo escrevemos a equação de Bellman como segue abaixo.

$$
\begin{aligned}
V_{2}\left(k_{t}\right)= & \max \left\{\log \left(k_{t}^{0}-k_{t+1}\right)+\beta V_{1} k_{t+1}\right\} \\
& \max \left\{\log \left(k_{t}^{0}-k_{t+1}\right)+\beta \theta \log _{t+1}\right\} .
\end{aligned}
$$

Com isso surge a implicação de que a regra de decisão $K_{t+1}=\alpha_{2}\left(k_{t}\right)=\gamma_{2} k_{t}^{\theta}$, onde $\gamma_{2}=\frac{\theta \beta}{1+\theta \beta}$. Analogamente, para o terceiro passo escrevemos.

$$
V_{3}\left(k_{t}\right)=\max \left\{\log \left(k_{t}^{\theta}-k_{t+1}\right)+\beta \theta(1+\beta \theta) \log \left(k_{t+1}\right)+D\right\}
$$

, onde D é uma constante.

Com isso, temos a regra de decisão de $k_{t+1}=\alpha_{3}\left(k_{t}\right)=\gamma_{3} k_{t}^{\theta}$, onde $\gamma_{3}=\frac{\theta \beta(1+\theta \beta)}{1+\theta \beta+\theta^{2} \beta^{2}}$. Então continuando desta maneira, no passo $n$ temos a função $k_{t+1}=\alpha_{n}\left(k_{t}\right)=\gamma_{n} k_{t}^{\theta}$, onde $\gamma_{n}$ é dada por,

$$
\gamma_{n}=\frac{\theta \beta\left(1+\theta \beta+\ldots+\theta^{n-1} \beta^{n-1}\right)}{1+\theta \beta+\ldots+\theta^{n} \beta^{n}}
$$

Na medida em que $n \rightarrow \infty$, vê-se que $\gamma_{n} \rightarrow \theta \beta$. Consequentemente, a regra de decisão converge para $k_{t+1}=\alpha\left(k_{t}\right)=\theta \beta k_{t}^{\theta}$. A função valor no passo n é dada por,

$$
V_{n}\left(k_{t}\right)=\theta\left(1+\theta \beta+\ldots+\theta^{n-1} \beta^{n-1}\right) \log k_{t}+D_{n}
$$

, onde D é uma constante.

Tratando o termo entre parênteses com a soma de uma progressão geométrica infinita 
temos a fórmmula fechada abaixo.

$$
V\left(k_{t}\right)=\frac{\theta}{1-\theta \beta} \log \left(k_{t}\right)+D_{0}
$$

, onde D é uma constante.

Pode-se afirmar que esta função valor satisfaz a equação de Bellman para o problema do horizonte infinito por meio da substituição em 2.10.

A equação de Euler neste exemplo pode ser escrita por

$$
\beta \theta k_{t+1}^{\theta-1}\left(k_{t}^{\theta}-k_{t-1}\right)=k_{t+1}^{\theta}-k_{t+2}
$$

A regra de decisão derivada acima, $k_{t+1}=\alpha\left(k_{t}\right)=\theta \beta k_{t}^{\theta}$, satisfaz a equação acima. Existem outras soluções para a equação de Euler, mas esta é a única solução que satisfaz também a condição de transversalidade. Por fim, neste exemplo pode-se mostrar diretamente a regra de decisão $k_{t+1}=\beta \theta k_{t}^{\theta}$ que $k \rightarrow k^{*}$ para qualquer condição inicial $k_{0}>0$. Aplicando a função $\log$ dos dois lados então $\log \left(k_{t+1}\right)=\log (\theta \beta)+\theta \log \left(k_{t}\right)$, e portanto $\log \left(k_{t}\right) \rightarrow \frac{1}{1-\theta} \log (\theta \beta)$. Daí temos que, $k^{*}=(\theta \beta)^{\frac{1}{1-\theta}}$.

Agora considerando um exemplo diferente com constante relativa de aversão ao risco $(\mathrm{CRRA}), u(c)=\frac{\left(c^{1-\alpha}-1\right)}{(1-\alpha)}$, onde $\alpha>0$, e crescimento tecnológico linear $y_{t}=A k_{t}$. Também seja $B=A+1-\gamma$ de modo que pode-se escrever $c_{t}=B K_{t}-k_{t+1}$, e assume-se que $\beta<\beta^{\frac{1}{\alpha-1}}$. A equação de Bellman é a que segue:

$$
V\left(k_{t}\right)=\max \left\{u\left(B k_{t}-k_{t+1}\right)+\beta V\left(k_{t+1}\right)\right\}
$$

A condição de primeira ordem é $u^{\prime}\left(\beta k_{t}-k_{t+1}\right)=\beta V^{\prime}\left(k_{t+1}\right)$. Inserindo $V^{\prime}$ temos a equação de Euler, $u^{\prime}\left(B k_{t}-k_{t+1}\right)^{-\alpha}=\beta B u^{\prime}\left(B k_{t+1}-k_{t+2}\right)$, ou reescrevendo no formato de uma função utilidade CRRA temos,

$$
\left(B k_{t}-k_{t+1}\right)^{-\alpha}=\beta B\left(B k_{t+1}-k_{t+2}\right)^{-\alpha}
$$

A condição necessária e suficiente para que uma sequência $k_{t}$ seja ótima é que satisfaça a equação de Euler e a condição de transversalidade. Isto é verdadeiro para que a sequência que iniciando de um dado ponto inicial, $k_{0}$, satisfaça $k_{t+1}=\gamma k_{t} \operatorname{com} \gamma=(\beta B)^{\frac{1}{\alpha}}$. Isto implicará que independentemente do valor de $k_{0}$ iniciamos com uma economia crescente à 
taxa fixa de $\gamma-1$, que depende de preferências e tecnologias, mas não da condição inicial. Continuando, inserimos $k_{t+1}=\gamma k_{t}$, na equação de Euler para produzir,

$$
(B-\gamma)^{-\alpha}=\beta B(B-\gamma)^{-\alpha} \gamma^{-\alpha}
$$

Isto é garantido, se e somente se, $\gamma=(\beta B)^{\frac{1}{\alpha}}$. Isto implica que $k_{t+1}=(\beta B)^{\frac{1}{\alpha} k^{t}}$, e $c_{t}(B-\gamma) k_{t}$. Note que $c_{t}>0$, e também $V<\infty$ sob as hipóteses adequadas de parâmetros, ou seja, se $\alpha<1$, então assume-se $B<\beta^{\frac{1}{\alpha-1}}$.

Além disso, esta mesma hipótese implica $\beta^{T} u^{\prime}\left(c_{t}\right) k_{T+1} \rightarrow 0$ na medida em que $T \rightarrow$ $\infty$, e assim assegura-se a condição de transversalidade.

Em particular para qualquer, $k_{0}$, temos $k_{t}=k_{0} \gamma^{t} \operatorname{com} \gamma=(\beta B)^{\frac{1}{\alpha}}$. Consequentemente, $B<\frac{1}{\beta}$ implica que $k_{t} \rightarrow 0$, e $\frac{1}{\beta}<B<\beta^{\frac{1}{\alpha-1}}$ implica que $k_{t}$ cresce sem atingir a restrição. Vale notar que se duas economias iniciam com diferentes valores de $k_{0}$, então tal diferença persistirá indefinidamente. Similarmente, se duas economias tem diferentes valores de $(\beta B)^{\frac{1}{\alpha}}$, então as mesmas crescerão ad eternum com diferentes taxas, ou seja, com diferença entre os seus respectivos níveis de renda, tornando-se mais distanciadas ao longo do tempo.

Por fim, para encerrar esta parte onde objetivamos descrever os modelos de crescimento econômico tradicionais, vale mencionar que alguns economistas, teóricos dos modelos macroeconômicos, trabalham a tempo contínuo, diferentemente do que foi apresentado, onde toda a formulação obedeceu a formalização de tempo discreto. Deste modo, muitos resultados na literatura macroeconômica são apresentados numa linguagem diferente daquela aqui apresentada.

De uma forma sintetizada pode-se esquematizar o modelo básico em tempo contínuo onde o problema do planejador central é dado por,

$$
\max \int_{0}^{\infty} e^{-\rho t} \mu\left(c_{t}\right) d t
$$

sujeito a $\dot{k}_{t}=f\left(k_{t}\right)-\gamma k_{t}-c_{t}$ e $k_{0}$ dado.

Passemos a derivar os resultados a tempo contínuo como limite dos resultados de tempo discreto. Como primeiro passo deste procedimento, seja $\Delta>0$ o comprimento dos períodos de tempo, de tal modo que os indivíduos consomem em tempos $t=0, \Delta, 2 \Delta, \ldots$ e a função utilidade dos indivíduos é, 


$$
\sum_{t} e^{-\rho t} \Delta \mu\left(c_{t}\right)
$$

Já que o período de tempo é de comprimento $\Delta$ pode-se escrevero fator de desconto como $\beta=\beta(\Delta)=e^{-\rho \Delta}$, e sendo o consumo fixo com $c_{t}$ entre $t$ e $t+\Delta$, a utilidade do período é $\Delta u\left(c_{t}\right)$. Vale notar que como $\Delta \rightarrow 0$ a somatória converge para a integral, assim como a produção e depreciação, o consumo também tem seu lugar em cada período, e a lei de movimentação do capital é $k_{t+\Delta}=k_{t}+\left[f\left(k_{t}\right)-\delta k_{t}-c_{t}\right] \Delta$.

Na medida em que $\Delta \rightarrow 0$, produzimos a lei de movimentação de capital em tempo contínuo. Para qualquer $\Delta>0$ fixo, o consumo satisfaz a identidade.

$$
c_{t}=k_{t}-k_{t+\Delta} \Delta+f\left(k_{t}\right)-\delta k_{t}
$$

O passo seguinte dentro da tarefa de determinar a solução do problema do planejador central em tempo contínuo, é inserir a identidade consumo dentro da função objetivo e diferenciar com respeito a variável de controle, $k_{t+\Delta}$, e assim obter a equação de Euler.

$$
-u^{\prime}\left(c_{t}\right)+e^{\rho \Delta} u^{\prime}\left(c_{t}+\Delta\right)\left[1+\Delta f^{\prime}\left(k_{t+\Delta}-\Delta \delta\right)\right]=0
$$

Rearranjando,

$$
\frac{-u^{\prime}(c t+\Delta)-u^{\prime}(c t)}{\delta}+u^{\prime}\left(c_{t}\right) \frac{1-e^{\rho \Delta}}{\Delta}+u^{\prime}\left(c_{t}+\Delta\right)\left[f^{\prime}\left(k_{t+\Delta}\right)-\delta\right]=0
$$

No limite, na medida em que $\Delta \rightarrow 0$, obtém-se a equação de Euler a tempo contínuo.

$$
u^{\prime \prime}\left(c_{t}\right) c_{t}=u^{\prime}\left(c_{t}\right)\left[\delta+\rho-f^{\prime}\left(k_{t+\Delta}\right)\right]
$$

Juntamente com a condição de transversalidadea solução está caracterizada. Consequentemente, o modelo de tempo contínuo é descrito pelo sistema dinâmico 2-dimensional, onde $A(c)=\frac{u^{\prime}(c)}{u^{\prime \prime}(c)}$. Dado $k_{0}$ qualquer solução para este sistema que satisfaça a condição de transversalidade é um equilíbrio. Para estudarmos soluções, primeiramente note que $k=0$, se e somente se, $c=f(k)-\delta k$ e $c=0$, e se somente se, $f^{\prime}(k)=\delta+\rho$ como a figura ilustra no plano $(k, c)$. Pode-se mostrar que existe um único não degenerado steady state que é o ponto de sela. Assim para qualquer $k_{0}>0$, existe um único $c_{0}$ no caminho de sela, 
de modo que iniciando em $\left(K_{0}, c_{0}\right),\left(K_{t}, c_{t}\right)$ segue até o steady state monotonicamente. O caminho de sela é a regra ótima de consumo, $c_{t}=c\left(k_{t}\right)$.

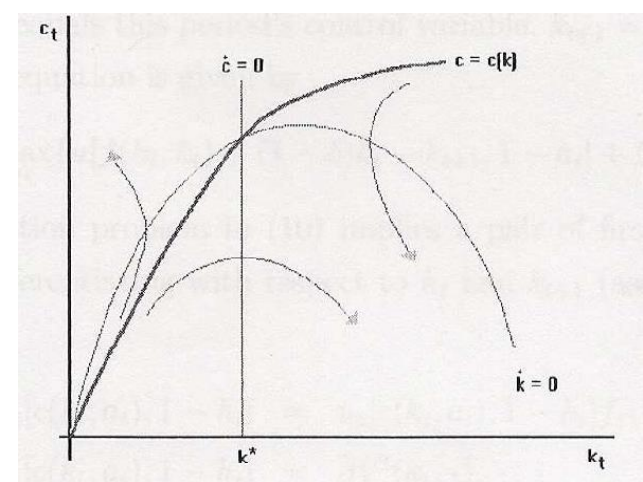

Figura 9: Modelo de Tempo Contínuo

\subsection{O modelo de Equilíbrio Dinâmico Estocástico - DSGE}

\subsubsection{Modelo Geral}

Inicialmente representaremos o ambiente econômico por um número grande de dois tipos de atores, famílias e firmas. As firmas são produtoras de um único produto que pode ser consumido e no qual se investe por meio de capital e trabalho. As firmas possuem capital e contratam trabalho das famílias. Assim a cada instante do tempo temos a interação de três tipos de mercado, o mercado de consumo, o mercado de trabalho, e o mercado financeiro que corresponde ao valor de mercado das firmas.

As famílias exibem preferências que são caracterizadas pela função utilidade, a seguir:

$$
E_{t} \sum_{\tau=t}^{\infty} \beta^{* \tau-t}\left(\log \left(c_{t}\right)-\theta \frac{h_{t}^{1+\psi}}{1+\psi}\right)
$$

onde $0<\beta^{*}<1$ é um fator de desconto constante, $c_{t}$ é o consumo no período t, $h_{t}$ é a fração do total de tempo disponível destinado a produção no tempo t, a desutilidade do trabalho $\theta>0$ e a elasticidade da oferta de trabalho é $\psi \geq 0$.

Assume-se que existe a figura do planejador central que é o responsável por determinar horas de trabalho, consumo e a acumulação de capital de modo a maximizar a utilidade das famílias, que estão sujeitas a seguinte restrição orçamentária 


$$
c_{t}+i_{t}=y_{t}
$$

onde $i_{t}$ é o investimento e $y_{t}$ é o produto. O investimento é utilizado para formar capital que é acumulado da seguinte forma:

$$
k_{t+1}=\exp \left(b_{t}\right) i_{t}+(1-\delta) k_{t}
$$

com $0<\delta<1$. Onde $\delta$ é a taxa de depreciação física constante e $b_{t}$ é uma choque que afeta o progresso tecnológico.

O produto $y_{t}$ é produzido por meio de capital e trabalho, contando com uma constante de tecnologia de retornos de escala constante, representado pela seguinte função de produção Cobb-Douglas:

$$
y_{t}=\exp \left(a_{t}\right) k_{t}^{\alpha} h_{t}^{1-\alpha}
$$

com $0<\alpha<1$, onde $a_{t}$ representa um choque estocástico de tecnologia. Assumimos que os choques de tecnologia são distribuídos com média zero, e apresentam persistência ao longo do tempo e correlação no período corrente. Consideramos o processo conjunto $\left(a_{t}, b_{t}\right)$ definido como

$$
\left[\begin{array}{l}
a_{t} \\
b_{t}
\end{array}\right]=\left[\begin{array}{ll}
\rho & \tau \\
\tau & \rho
\end{array}\right]\left[\begin{array}{l}
a_{t-1} \\
b_{t-1}
\end{array}\right]+\left[\begin{array}{c}
\epsilon_{t} \\
\nu_{t}
\end{array}\right]
$$

onde $|\rho+\tau|<1$ e $|\rho-\tau|<1$ por conta da estacionariedade, e, adicionalmente:

$$
\begin{gathered}
E\left(\epsilon_{t}\right)=0, \\
E\left(\nu_{t}\right)=0, \\
E\left(\epsilon_{t} \epsilon_{s}\right)= \begin{cases}\sigma_{\epsilon}^{2} & \text { se } t=s \\
0 & \text { se } t \neq s\end{cases} \\
E\left(\nu_{t} \nu_{s}\right)= \begin{cases}\sigma_{\nu}^{2} & \text { se } t=s \\
0 & \text { se } t \neq s\end{cases}
\end{gathered}
$$




$$
E\left(\epsilon_{t} \nu_{s}\right)= \begin{cases}\varphi \sigma_{\epsilon} \sigma_{\nu} & \text { se } t=s \\ 0 & \text { se } t \neq s\end{cases}
$$

\subsubsection{Equilíbrio Dinâmico do modelo geral}

A determinação do equilíbrio para esta economia passa por resolver o problema do consumidor, que é determinar as sequências $y_{t}, c_{t}, i_{t}, h_{t}, k_{t+1}$ que maximizam a função utilidade do consumidor, exposta em 2.36, conforme definido pelo planejador central sujeitando-se ao conjunto de restrições apresentadas nas equações: 2.37 - restrição orçamentária, 2.38 - regra de acumulação de capital, 2.39 - função de produção e 2.40 - processo gerador de choques - para todo $t=0,1,2, \ldots$ que conduzem à trajetória de equilíbrio da economia.

O equilíbrio dinâmico da economia que estamos representando é obtido a partir da condição de primeira ordem de otimalidade como segue abaixo:

$$
\begin{aligned}
& c_{t} \theta h_{t}^{1+\psi}=(1-\alpha) y_{t} \\
& \beta E_{t}\left[\left(\frac{\exp \left(b_{t}\right) c_{t}}{\exp \left(b_{t+1}\right) c_{t+1}}\right)\left(\exp \left(b_{t+1}\right) \alpha \frac{y_{t+1}}{k_{t+1}}+1-\delta\right)\right]=1 \\
& y_{t}=\exp \left(a_{t}\right) k_{t}^{\alpha} h_{t}^{1-\alpha} \\
& k_{t+1}=\exp \left(b_{t}\right)\left(y_{t}-c t\right)+(1-\delta) k_{t} \\
& a_{t}=\rho a_{t-1}+\tau b_{t-1}+\epsilon t \\
& b_{t}=\tau a_{t-1}+\rho b_{t-1}+\nu t
\end{aligned}
$$

Acerca das equações acima vale notar que a primeira equação retrata a função de oferta de trabalho. Nesta equação notamos a condição de eficiência intratemporal que iguala a taxa marginal de substituição entre consumo e lazer ao produto marginal do trabalho. Enquanto que a segunda equação é a equação de consumo intertemporal de Euler onde verifica-se a condição de eficiência intertemporal que é a igualdade da taxa marginal de substituição intertemporal ao produto marginal do capital.

O conjunto de equações acima formam um sistema de seis equações estocásticas de 
diferenças não-lineares de um modelo de seis variáveis: $y_{t}, c_{t}, i_{t}, h_{t}, k_{t}$ e $a_{t}$.

\subsubsection{Solução do modelo geral}

A fim de apresentar uma solução geral para os modelos DSGE, conforme apresentado no Anexo A deste documento uma implementação de código via o engine de cálculo Dynare. Tal ferramenta funciona com um motor de cálculo customizada para o enfoque do DSGE, e pode ser acessada em http://www.dynare.org/download. De forma mais direta, trata-se de um pré-processador que acessa uma coleção de rotinas do Matlab, como pode ser visto no esquema abaixo.

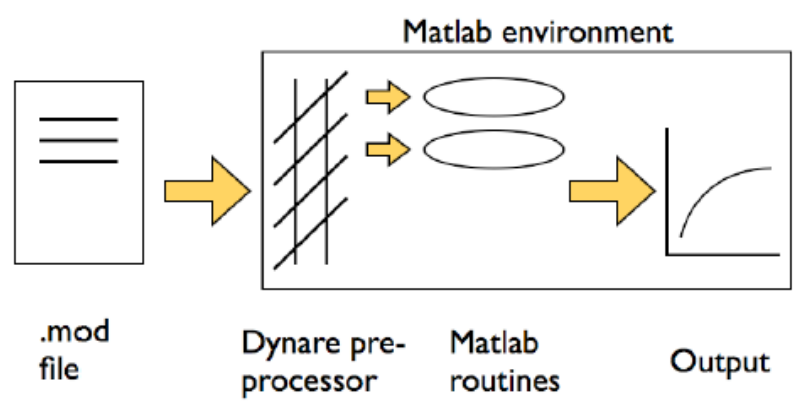

Figura 10: Ambiente de funcionamento Dynare

De forma suscinta pode-se dizer que por meio do motor de cálculo do Dynare é possível determinar: (a) o steady state do modelo, (b) a solução para modelos determinísticos, (c) determinar a aproximação de primeira ordem para os modelos estocásticos, (d) estimar os parâmetros do modelo DSGE por meio de maxima verossimilhança como pelo enfoque bayesiano, e (e) a política ótima. 


\section{O MODELO DSGE DE SMETS-WOUTERS}

O modelo de Smets-Wouters apresentado em 2003 sob o formato de um artigo, com o título - An estimated dynamic stochastic general equilibrium model of the euro area. (Journal of the European Economic Association., vol.1 (5), p.1123-1175, 2003) - é, de fato, um sistema não linear de equações na forma de um modelo dinâmico estocástico de equilíbrio geral (DSGE) que busca descrever uma economia caracterizando-a a partir de princípios básicos de funcionamento econômico. O modelo básico trabalha com séries temporais de 7 variáveis econonômicas, tais como: produto interno bruto, preços, salários, horas trabalhadas, taxa de juros, consumo e investimento.

Ao passo que a forma de trabalho tradicional da macroeconomia tem sido criar modelos de regressão "grandes" com razoável fundamentação empírica, o enfoque dos modelos DSGE passa a ser focar em "pequenos" modelos originados de derivação teórica. Sendo, portanto, um combinação de rigor normativo e parcimônia, o que passa a ser como um apelo do enfoque DSGE.

Atualmente, os modelos DSGE são frequentemente usados para fins de análise de política monetária. Na literatura econômica modelos macroeconômicos que são construídos de forma microfundamentada, seguindo a metodologia de equilíbrio geral. Esta classe de modelos, grosso modo, evoluiu para a classe de modelos denominada de ciclos econômicos reais, ou do original em inglês, Real Business Cycle models (RBC) os quais surgiram na década de 1980.

Os modelos de ciclos econômicos reais sustentam em sua arquitetura que as flutuações, de forma geral, na atividade econômica devem-se a choques econômicos, como por exemplo, mudanças na taxa de progresso tecnológico por meio da introdução de novos padrões de tecnologia.

De posse de um modelo DSGE parcimonioso, a forma linearizada de um modelo DSGE pode ser montada como um modelo VAR (Vector Autoregressive) que pode ser manuseado através de métodos oriundos da análise de séries temporais. Trata-se de uma 
forma irrestrita do modelo VAR que examinaremos na próxima seção deste trabalho.

Para fins de propósito de ilustração, adicionaremos a série de tempo, desemprego, pois embora não seja parte do estrutura básica do modelo de Smets-Wouters, poder medir a taxa de desemprego implica em realizar uma medição acerca da saúde de uma economia.

Embora o modelo original dos autores Smets-Wouters tenha sido desenvolvido para o agregado de países da Europa, neste trabalho aplicaremo a economia Americana. Neste ponto da aplicação, um obstáculo para a não aplicação, por ora, para a economia Brasileira, foi o fato das séries temporais econômicas brasileiras serem mais curtas, e permeadas de choques econômicos, o que nos faria utilizar tais séries temporais a partir da introdução do plano Real, o qual iniciou-se em 1994. Teríamos assim um histórico máximo de 20 anos, isto sem contar que o início do período de estabilização econômica que iniciou-se em 1994 perderou pelos anos seguintes. Vale abrir uma pequena digressão para notar que a literatura registra algumas iniciativas promovidas, até o momento sob o formato de working paper, patrocinadas pelo BACEN para a implementação de um modelo DSGE adaptado para a realidade brasileira. Tal iniciativa é conhecida pelo acrônimo SAMBA, tornada pública em 2011 através da publicação por Castro, M.R et al do artigo SAMBA: Stocasthic Analytical Model with a Bayesian Approach. 


\subsection{Base de Dados}

Ao longo deste trabalho as séries temporais tanto econômicas quanto financeiras dos indicadores com as quais trabalharemos serão referentes a economia Americana, considerando o período de 1950 até 2011.

\begin{tabular}{|l|}
\hline Gasto com compensação paga aos trabalhadores em bilhões de dólares \\
\hline Taxa de Inflação ao Consumidor \\
\hline Taxa de básica de juros pós-fixada \\
\hline Gasto governamental e investimento \\
\hline PIB em bilhões de dólares \\
\hline PIB deflacionado \\
\hline Investimento doméstico em bilhões de dólares \\
\hline Taxa de juros dos títulos soberanos de 10 anos de maturidade \\
\hline Horas trabalhadas no Setor não-industrial \\
\hline Oferta monetária base M1 \\
\hline Oferta monetária base M2 \\
\hline Consumo pessoal medido em bilhões de dólares \\
\hline Taxa de juros soberana de 3 meses
\end{tabular}

Tabela 2: Base de dados das séries temporais

\subsection{Ciclos Econômicos}

De modo a examinar a interposição entre ciclos econômicos e o modelo DSGE representativo da economia americana que se pretende construir, fizemos a inclusão das datas dos pontos de máximos e mínimos dos ciclos econômicos.

\subsection{Especificação do Modelo DSGE}

O vetor autoregressivo (VAR) é uma ferramenta de utilização frequente nos estudos em que deseja medir a interação entre variáveis econômicas fundamentais de um modelo econômico, ou seja, é uma ferramenta amplamente disseminada para se estudar a relação e/ou comportamento entre as variáveis chave de um sistema econômico.

Grosso modo, pode-se dizer que é possível aplicar a metodologia do VAR aos dados observados na realidade econômica, e, também aos dados gerados pelo modelo e compará-los por meio da trajetória de suas funções impulso-resposta. Assim espera-se que a trajetória das funções impulso-resposta estimada a partir dos dados gerados pelo modelo estejam circunscritas às bandas do intervalo de confiança das trajetórias observadas pela 
função impulso-resposta obtidas a partir dos dados coletados da realidade econômica. Em se verificando tal premissa temos evidências que nos sugerem que o modelo DSGE especificado reproduz de forma assemelhada o comportamento do mundo econômico real.

Vetores autoregressivos (VARs) ou Vetores autoregressivos estruturais (SVARs) - ou seja, modelos VAR que contam com uma configuração de um conjunto mínimo de restrições que permitem traçar a o efeito de pelo menos alguns choques estruturais - são utilizados após a estimação para comparar a função impulso-resposta dos choques implícitos nos modelos estruturais com os resultados obtidos a partir da interpretação tradicional dos dados.

De forma geral os modelos econômicos são escritos como função de diversas variáveis, portanto, a utilização de modelos univariados mostra-se muito limitada para o objetivo de refletir um modelo econômico. Os modelos VAR apresentam como ponto forte a capacidade de expressar a robustez econômica da realidade associada a adequada estimação dos parâmetros do modelo.

Vale notar que uma atenção deve ser dado ao fato de que modelos construídos no ambiente da metodologia VAR apresentam um conjunto de restrições, e, o estudo de tais restrições, e, a utilização das mesmas é o objetivo embutido na metodologia VAR.

Um modelo VAR pode ser representado por um modelo regressivo de ordem $p$ de um vetor de n-variáveis endógenas, $X_{t}$, que são ligados por uma matriz $A$ como segue:

$$
A X_{t}=B_{0}+\sum_{i=1}^{p} B_{i} X_{t-i}+B \epsilon_{t}
$$

onde

- A é uma matriz $n \times n$ que define restrições contemporâneas entre as variáveis que constituem o vetor $n \times 1, X_{t}$;

- $B_{0}$ é um vetor de constante $n \times 1$;

- $B_{i}$ são matrizes $n \times n$;

- B é uma matriz diagonal de devio padrão $n \times n$; 
- $\epsilon$ é uma perturbação não correlacionada para cada uma dos vetores simultâneos temporalmente $n \times 1$, a saber: $\epsilon_{t} \approx i . i . d .\left(\mathbf{0}, I_{n}\right)$

A equação acima expressa a relação entre variáveis endógenas de um modelo econômico teórico estruturado, também chamada de forma estrutural. Os choques $\epsilon_{t}$ são denominados choques estruturais porque individualmente impactam cada um das variáveis exógenas.

Os choques estruturais são considerados independentes entre si porque a interelação entre choques é indiretamente capturada pela matriz A. Portanto, a independência dos choques ganha sustentação sem perda de generalidade. Assim as variáveis endógenas deste modelo são geralmente estimadas na sua forma reduzida, isto é, estimadas com o seguinte modelo.

$$
\begin{gathered}
X_{t}=A^{-1} B_{0}+\sum_{i=1}^{p} A^{-1} B_{i} X_{t-i}+A^{-1} B \epsilon_{t} \\
=\Phi_{0}+\sum_{i=1}^{p} \Phi_{i} X_{t-i}+e_{t},
\end{gathered}
$$

where

$$
\Phi_{i} \equiv A^{-1} B_{i}, i=0,1, \ldots, p \quad B \epsilon_{t} \equiv A e_{t}
$$

Para ilustrar, um exemplo de modelo bivariado de ordem 1 é mostrado abaixo.

$$
\begin{aligned}
& y_{t}=b_{10}-a_{12} z_{t}+b_{11} y_{t-1}+b_{12} z_{t-1}+\sigma_{y} \epsilon_{y t} \\
& z_{t}=b_{20}-a_{21} y_{t}+b_{21} y_{t-1}+b_{22} z_{t-1}+\sigma_{z} \epsilon_{z t}
\end{aligned}
$$

Trata-se de uma especificação razoavelmente adequada, pois as variáveis são mutuamente influenciadas umas pelas outras, ambas contemporâneamente e pelos seus valores defasados. Este modelo pode ser estimado diretamente, já que ambas as variáveis contemporâneas $z_{t}$ e $y_{t}$ são individualmente correlacionadas com erros $\epsilon_{y t}$ ou $\epsilon_{z t}$, respectivamente. Isto ocorre porque cada uma das variáveis depende das demais contemporâneamente (efeito feedback). Estes efeitos ocorrem porque $\epsilon_{y t}$ e $\epsilon_{z t}$ afetam um ao outro. O objetivo da metodologia VAR é desenvolver técnicas para evitar este tipo de problema, visando determinar a trajetória de interesse antes do choque nos erros, em 
outras palavras, choques estruturais.

As hipóteses para este modelo são:

- $y_{t}$ e $z_{t}$ são ambas estacionárias;

- $\epsilon_{y t} \approx W N(0,1)$ and $\epsilon_{z t} \approx W N(0,1)$

- $\epsilon_{y t}$ and $\epsilon_{z t}$ são ortogonais implicando que $\operatorname{Cov}\left(\epsilon_{y t}, \epsilon_{z t}\right)=0$.

O modelo apresentado é definido na sua forma estrutural e pode ser escrito em forma matricial.

$$
A X_{t}=B_{0}+B_{1} X_{t-1}+B \epsilon_{t}
$$

A forma reduzida deste modelo é dada por:

$$
\begin{aligned}
X_{t} & =\Phi_{0}+\Phi_{1} X_{t-1}+e_{t} \\
\Phi_{0} & \equiv A^{-1} B_{0} ; \quad \Phi_{1} \equiv A^{-1} B_{1} \\
A e_{t} & \equiv B \epsilon_{t}
\end{aligned}
$$

A forma generalizada do VAR de ordem $(p)$ com variáveis endógenas é feita pela seguinte representação econométrica.

$$
X_{t}=\Phi_{0}+\sum_{i=1}^{p} \Phi_{i} X_{t-i}+G Z_{t}+e_{t}
$$

onde:

$X_{t}$ é um vetor $\mathrm{n} \times 1$;

$\mathrm{G}$ é uma matriz de coeficientes $\mathrm{n} \times \mathrm{g}$;

$Z_{t}$ é um vetor $\mathrm{g} \times 1$ de variáveis exógenas que pode incluir variáveis determinísticas.

O modelo desenvolvido neste trabalho é um modelo VAR irrestrito na forma.

$$
y_{t}=a+\sum_{i=1}^{p} A_{i} y_{t-i}+W_{t}
$$

com, 


$$
\begin{aligned}
& \qquad y_{t}=\left(y_{1} ; \ldots ; y_{8}\right) \\
& y_{1}=P I B \ldots ; y_{2}=P I B D e \text { flacionado } \ldots ; y_{3}=S A L A R I O S \ldots ; y_{4}=H O R A S \ldots ; \\
& y_{5}=J U R O S \ldots ; y_{6}=C O N S \ldots ; y_{7}=I N V E S T \ldots ; y_{8}=D E S E M P R E G O \ldots \\
& \text { e, } \\
& W_{t} \approx N(0, Q) \\
& \text { em que, W - representa dados referentes a inovação. }
\end{aligned}
$$

\subsection{Ajuste do modelo}

Nesta seção buscamos responder a questão sobre como define-se a ordem de defasagem ótima de um modelo VAR, e quais critérios poderiam ser usados nesta tarefa.

Trata-se de uma tarefa árdua, pois de fato, a defasagem ótima para se obter 'resíduos brancos' relacionados à primeira variável endógena, não é a mesma ordem de dafasagem para se obter 'resíduos brancos' para a segunda variável endógena. Logo, a regra é usar tanto quanto possível a ordem de defasagens para obter resíduos brancos para todas as variáveis endógenas. Na prática é praticamente improvável porque há uma alta probabilidade que a função de autocorrelação para cada variável seja diferente de zero mesmo que a ordem de defasagem seja elevada. Isto ocorre porque 5 por cento dos valores estimados estariam fora do intervalo de confiança. Por outro lado, usando uma ordem de defasagem elevada em um modelo muito complexo no qual estima-se muitos coeficientes cruzados torna o poder do teste estatístivo muito fraco. Em síntese, bom senso e parcimônia devem ser usados neste processo.

Baseado no que discutimos acima, o primeiro passo em nossa análise é determinar o número 'ótimo' da ordem de defasagem baseado na estatística do Critério de Informação de Akaike (AIC). Construímos um modelo com até ordem de defasagem 7, ou seja, 7 trimestres e analisamos a performance da estatística de Akaike, sendo que o valor mínimo da estatística AIC será adotado como o número de defasagens ótimo.

O caso geral do AIC é definido por 


$$
A I C=2 k-2 \ln (L)
$$

, onde $k$ é o número de parâmetros no modelo estatístico e $L$ é o valor maximizado da função de verossililhança do modelo estimado.

Dado um conjunto de modelos candidato para os dados utilizados, o modelo escolhido é aquele que atende ao critério de valor AIC mínimo. Consequentemente a estatística AIC não recompensa somente a qualidade do ajuste 'goodness of fit', mas também inclui uma penalidade que é uma função crescente do número de parâmetros a serem estimados. Tal penalidade claramente desencoraja o overfitting - o aumento do número de parâmetros livres para aperfeiçoar a qualidade do ajuste, independentemente do número de parâmetros livres no processo gerador de dados. Isto porque, o critério de AIC superestima assintoticamente a ordem do VAR com probabilidade positiva, ao passo que outros critérios como por exemplo, o BIC (Critério Bayesiano de Informação), estimam a ordem consistentemente sob hipóteses bem gerais.

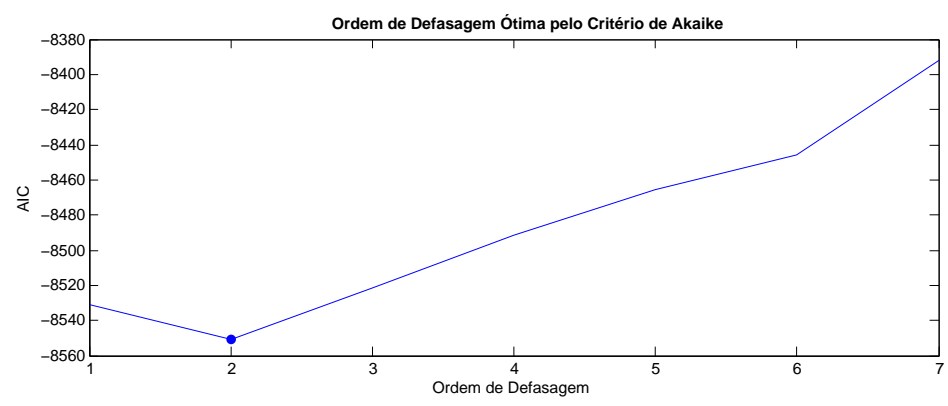

Figura 11: Estatística de Akaike 


\subsection{Avaliação da Precisão das Projeções do Modelo}

Determinado o número ótimo de defasagens regressivas, 'lags', o próximo passo é determinar a precisão de modelo construído. Para fazer isto, implementamos uma simulação de Monte carlo com uma amostragem de 500 amostras de trajetórias para cada ano desde 1975 até o mais recente ano.

Definido o número ótimo de defasagens do operador autoregressivo o passo seguinte é definir a acurácia de previsão do modelo. Para obtermos tal objetivo produzimos uma simulação de Monte Carlo com uma amostragem de possíveis trajetórias para cada ano para as variáveis consideradas. Na sequência, dada as amostras definidas para cada ano, estimamos o erro quadrátivo médio (RMSE) entre realizações e projeções subsequentes ao longo do horizonte de tempo. Para esta análise, a projeção do horizonte projetado foi de 1 ano.

A projeção dos erros é visualizado no gráfico 3.5. Em cada subgráfico a linha azul representa o RMSE médio associado a cada data juntamente temos a média da amostra (linha verde) e as bandas de desvio padrão (linhas vermelhas pontilhadas) destes erros ao longo das datas. O valor 1 no gráfico corresponde a 1 por cento do erro de previsão. A utilidade do desvio padrão é proporcionar uma análise qualitativa onde tem-se um guia para verificar a variação dos erros de previsão.

Examinando os subgráficos podemos ser conduzidos a fazer certas inferências sobre o relacionamento das inferências econômicas. Plotamos 8 subgráficos, e em 4 deles um novo comportamento tem sido notado após a crise de crédito, subprime, vivido pelo sistema bancário em 2008.

O subgráfico Preços nos mostar que após a crise do subprime o nível de preços tendo a ser menor do que tem sido até 2008, e este resultado poderia nos conduzir para uma conjutura que a demanda que se reduziu pode estar começando a retomar.

Analisando o subgráfico Horas trabalhadas a inferência que pode ser sugerida pela é que crise fiscal destruiu muitas horas trabalhadas e provavelmente a taxa de desemprevo deve aumentar.

A redução da taxa de juros Taxa de juros básica de curto prazo nos mostra que o Federal Reserve implementou uma estratégia de diminuição do custo da taxa de juros por meio da compra de ativos, carteiras de empréstimos, dos bancos como um procedimento para estimular a recuperação da economia. 

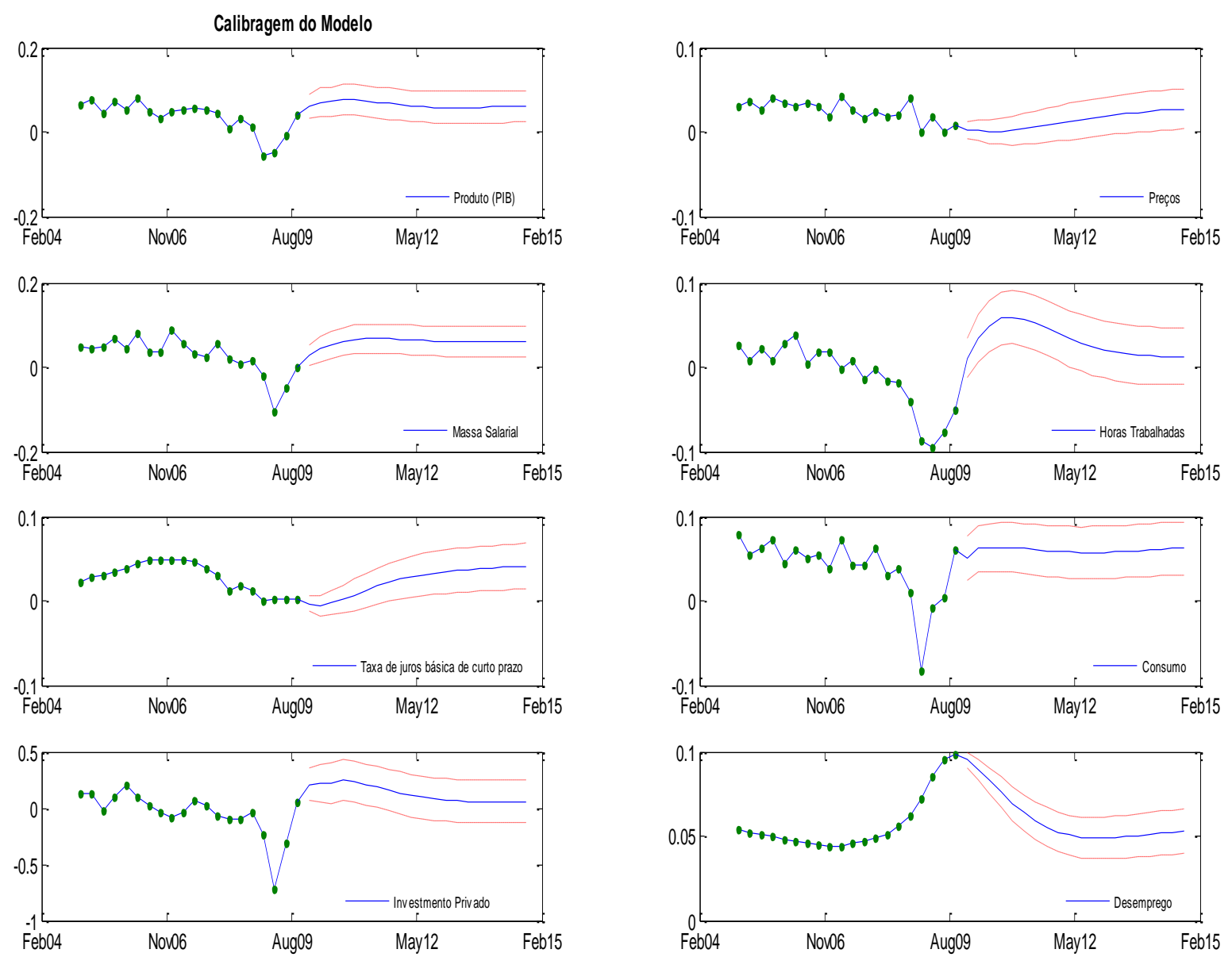

Figura 12: Calibragem do Modelo

A política de esforços para fazer a economia continuar rodando no nível de emprego natural, Desemprego, nos mostra que uma tendência de desemprego de longo prazo viria a ser experimentada pelos Estados Unidos e demandará esforços dos formuladores de política econômica.

Neste ponto uma questão importante deve ser colocada, segundo a persepctiva do modelo de Smets-Wounters a oferta monetária não é observada, tão pouco o que poderia acontecer se adicionássemos a oferta monetária. A resposta é interessante e pode ser verificado que não há melhora da previsão com a introdução da oferta monetária. 


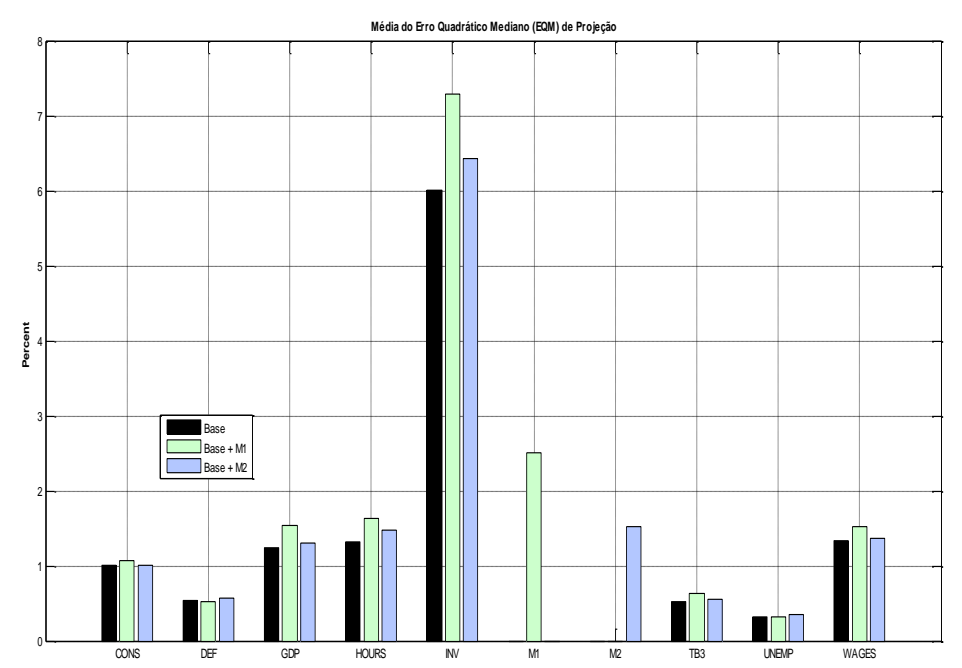

Figura 13: Desvio Padrão da Projeção do Erro das Variáveis Estimadas

\subsection{Análise Impulso-Resposta}

A análise impulso-resposta nos permitem ver quais variáveis apresentam dinâmica mais interessante na sua resposta a um choque ou ainda se não se mostram convergentes no estado de equilíbrio de longo prazo. Neste exercício podemos ver quais variáveis têm uma dinâmica mais voláteis ou oscilatórios.
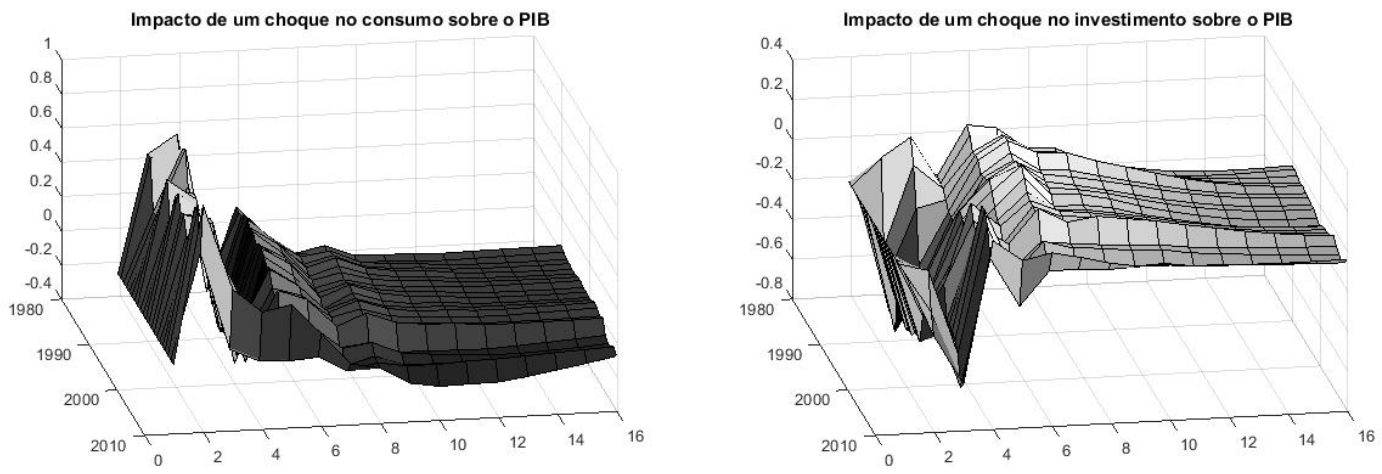

Figura 14: Impulso Resposta a um choque 


\subsection{Previsão do PIB}

A análise final é a previsão do PIB rela baseda no modelo calibrado até o momento. O valor projetado é comparado com a tendência de longo prazo baseado nos dados de PIB real dos últimos 30 anos.

No caso da curva de previsão iniciar abaixo e depois aproximar-se da linda de tendência, isto implica numa expansão - e recuperação - uma vez que o crescimento do PIB deveria ultrapassar a tendência de crescimento e retornar para a linha de tendência. Observando o gráfico que descreve a previsão do PIB é possível identificar que as previsões de 2011 à frente nos sugeririam um recuperação para os próximos anos.

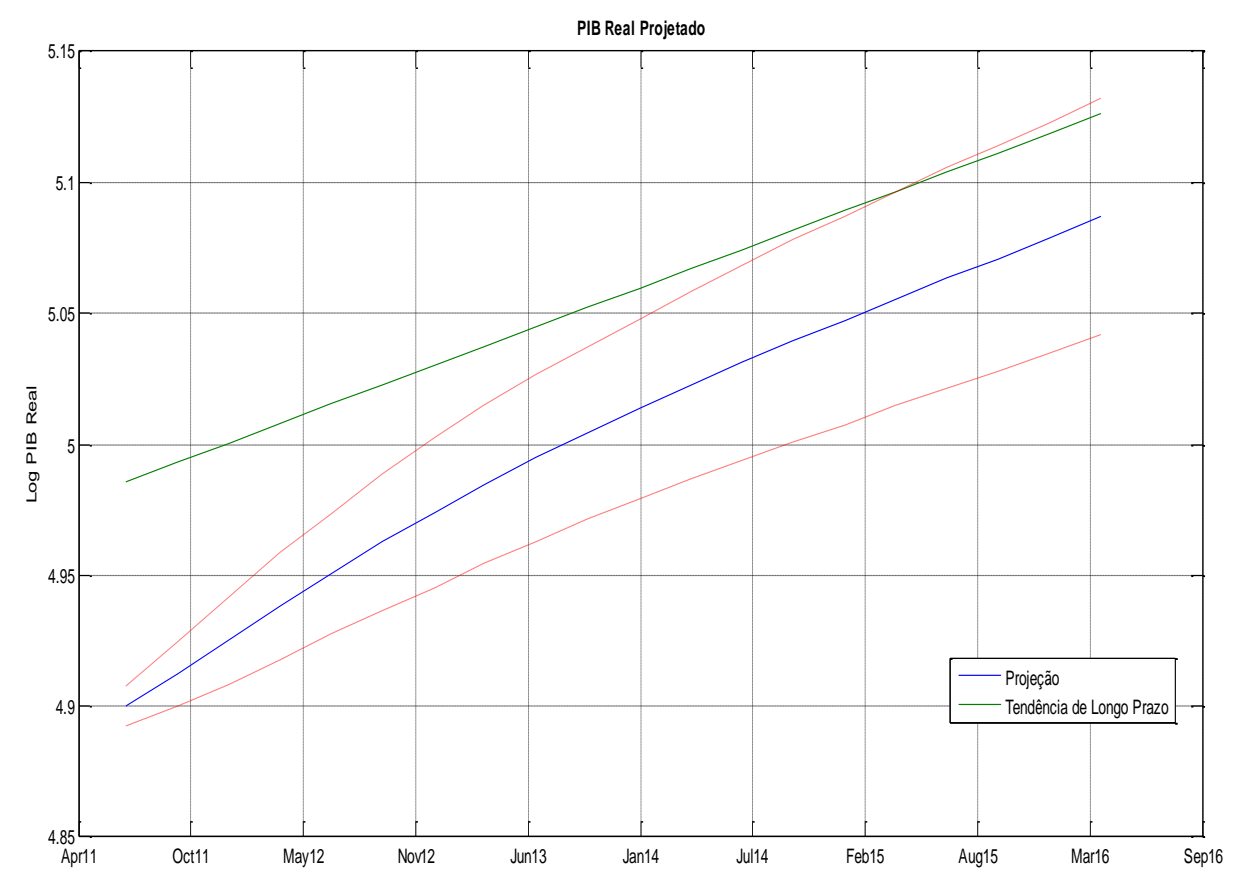

Figura 15: Estimativa de Crescimento do PIB 


\section{CONCLUSÕES E TRABALHOS FUTUROS}

Como ponto central que governa as discussões em torno da adoção dos modelos DSGE tem-se a sua sensibilidade à escolha de parâmetros. Assim a depender de quão apurada sejam tais parâmetros melhor será o resultado final da previsão. Outro ponto relevante é a capacidade do modelo em reproduzir as respostas empíricas, verificadas por meio da análise econométrica dos dados, este é um grande ponto de atenção dos modelos estocásticos de equilíbrio geral, e nota-se na literatura que vem sendo recentemente tratado com a introdução de fricções financeiras nos modelos. Por fim existe uma grande sensibilidade dos resultados gerais à suposição de diferentes regras fiscais, o que é relevante para economias com sistema fiscal imperfeito.

Do exercício realizado tomando-se em conta o paper de Smets-Wouters (2003), aplicado inicialmente à Europa, notamos que a forma linearizada do modelo por meio do enfoque na utilização do VAR (Vector Autoregressive) permitiu a realização de projeções com razoável acurácia para o crescimento econômico, medido pelo PIB, de modo que observa-se para este indicador uma projeção que é aderente à tendência de longo prazo.

Por fim, vale ressaltar que a adequada utilização dos resultados produzidos pelo modelo DSGE é um requisito de qualquer analista de política econômica. É possível afirmar que mesmo banco centrais de países com economias desenvolvidas, e, portanto com menores graus de interferências e imperfeições, não se apoiam somente nos resultados de seus modelos DSGE para deliberar qual caminho a seguir, na verdade, os resultados dos modelos DSGE atendem bem ao quesito de complementariedade, na medida, em que servem a tarefa de sustentar ou refutar uma política econômica. 


\section{REFERÊNCIAS}

BLANCHARD, O. The State of Macro. NBER. Working Paper, No. 14259, Agosto 2008.

BUENO, R. De Losso da S. Econometria de Séries Temporais.

CANOVA, F. Sensitivity Analysis and Model Evaluation in Simulated Dynamic General Equilibrium Economies. International Economic Review.

CHOW, G. C. Analysis and Control of Dynamic Systems. New York: Wiley, 1975.

CHRISTIANO, L.J. Linear-Quadratic Approximation and Value-Function Iteration: A Comparison. American Statistical Association.

CHRISTIANO, L. J.; EICHENBAUM, M.; VIGFUSSON, R.; KEHOE, P.; WATSON, M. W. Assessing Structural VARs. Chicago Journals.

DAY, R.H. Irregular growth cycles. American Economic Review, n. 72, 406-414, 1982.

DAY, R.H.; SHAFFER, W. Ergodic Fluctutations in deterministic economic models Journal of Economic Behavior and Organization. vol.8. 339-361. 1987.

DEJONG, D. ; CHETAN, D. Structural Macroeconometrics.

DIB, A. An estimated Canadian DSGE model with nominal and real rigiditie. The Canadian Journal of Economics.

DICECIO,R.; NELSON, E. An Estimated DSGE Model for the United Kingdom. Federal Reserve of St. Louis Review.

DYNARE; Reference Manual, Version 4, Dynare Working Papers, 1, CEPREMAP. ENDERS, W. Applied Econometrics Time Series. Wiley Series, 1995.

HAMILTON, J. Time Series Analysis. New Jersey: Princeton University Press, 1994.

HARVEY, A. C. Time Series Models. Harvester Wheatsheaf, 1993. 
LIM, G.C.; McNELIS, P. D. Computational Macroeconomics for the Open Economy.

LYNCH, S. Dynamical Systems with applications using Matlab.

SANTOS, M.S.; PERALTA-ALVA, A. Accuracy of Simulations for Stochastic Dynamic Models. Econometrica.

MATLAB - Technical Documents, The MathWorks, Inc.

SAMBA Stocasthic Analytical Model with Bayesian Approach, BACEN, 2011.

SIMS, C. Models and Their Uses. American Journal of Agricultural Economics.

SMETS, F.; WOUNTERS, R. An estimated dynamic stochastic general equilibrium model of the euro area. Journal of the European Economic Association., vol.1 (5), p.1123-1175, 2003.

TRAMONTANA, F., GARDINI, L., AGLIARI, A. Endogenous cycles in discontinuous growth models. Mathematics and Computers in Simulation, n. 81, 1625-1639.

WIELAND, V. Model Comparison and Robustness: A Proposal for Policy Analysis after the Financial Crisis. 


\section{ANEXO A - IMPLEMENTAÇÃO DO CÓDIGO DO DYNARE}

O primeiro passo consiste em declarar no preâmbulo as variáveis exógenas, as variáveis endógenas, os parâmetros e os valores dos parâmetros do modelo.

- Variáveis Endógenas

var $y, c, k, h, a, b$;

Refere-se a especificação das variáveis endógenas do modelo, já que temos produto (y), consumo (c), capital (k), horas de trabalho (h) e os dois choques (a, b).

- Variáveis Exógenas

varexo e, u;

Refere-se a especificação das variáveis exógenas do modelo, ou seja, os choques de inovação. Sendo que (e) corresponde aos choques não incorporados, e (u) corresponde a inovações de choques incorporados.

- Lista de parâmetros do modelo DSGE

A seguir apresentamos a lista de parâmetros no modelo que estamos estudando.

beta fator de desconto

alpha elasticidade do capital na função de produção

delta taxa de deprecição

theta desutilidade do parâmetro de trabalho

psi elasticidade da oferta de trabalho

rho persistência

tau persistência cruzada 
- Atribuição de valores dos parâmetros do modelo

$$
\begin{aligned}
& \text { alpha }=0.36 ; \\
& \text { rho }=0.95 ; \\
& \text { tau }=0.025 ; \\
& \text { bet } a=0.99 ; \\
& \text { delta }=0.025 ; \\
& \text { psi }=0 ; \\
& \text { theta }=2.95 ;
\end{aligned}
$$

A seguir passamos para a declaração do código, valores de inicialização e os choques de inovação e respectiva matriz de variância-covariância.

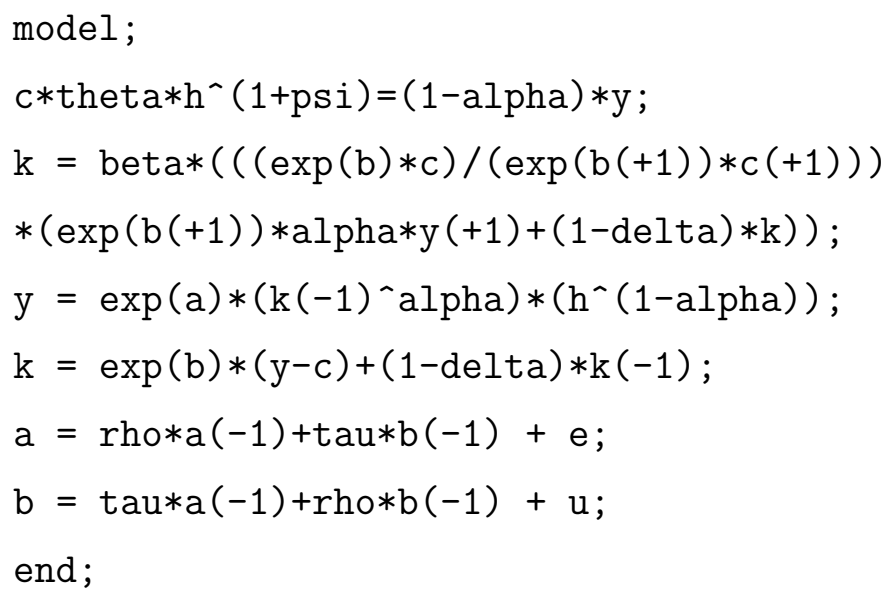


var e, u $=$ phi*0.009*0.009;

end;

stoch_simul; 